\title{
DISEÑO ROBUSTO DE LA CADENA DE SUMINISTRO HORTÍCOLA: CASO DE ESTUDIO HORTICULTORES DEL MUNICIPIO DE CAJICÁ
}

\author{
Dairo Steven Muñoz-Pinzón', Mónica Alejandra Jiménez-Méndez¹, Andrés Polo-Roa', Karen Tatiana \\ Valencia-Rivero ${ }^{1}$
}

${ }^{1}$ Fundación Universitaria Agraria ce Colombia.

Correo de correspondencia: dairo.munoz@outlook.com

\begin{abstract}
RESUMEN
En el presente documento se presenta una metodología para realizar el diseño robusto de cadenas de suministro hortícola, esto empleando modelación matemática y metodología propuesta por (Tordecilla Madera \& Leonardo, 2012). Se diseñó un modelo matemático que permita el diseño de la cadena hortícola y luego se evaluaron 7 configuraciones de cadena de suministro para determinar la más robusta, esto a partir de la evaluación de utilidades, emisiones de CO2 y medidas de desempeño operacional como la utilización de diferentes locaciones. El modelo empleado para el estudio es un modelo de Programación Lineal Entera Mixta (MIP) que permitió establecer la configuración de la cadena hortícola, apertura de centros de cross docking, centros de distribución de evaluación de vehículos para realizar el abastecimiento. Se estableció la configuración más robusta de la cadena hortícola, para lo cual, se definieron las locaciones que más convenía habilitar, así como los vehículos por adquirir para cuando existen niveles de oferta bajo, medio y alto de hortalizas, esto involucrando variaciones en los precios de venta en los diferentes eslabones de la cadena.
\end{abstract}

Palabras clave: cadena de suministro, hortalizas, horticultor, robustez.

Recibido: 19 de Febrero de 2020. Aceptado: 18 de Mayo de 2020

Received: February 18, 2020. Accepted: May 18, 2020

\section{ROBUST DESIGN OF THE HORTICOLA SUPPLY CHAIN: CASE STUDY OF HORTICULTORS OF THE MUNICIPALITY OF CAJICÁ}

\begin{abstract}
This document presents a methodology for the robust design of horticultural supply chains, this using mathematical modeling and methodology proposed by (Tordecilla Madera \& Leonardo, 2012). A mathematical model that allows the design of the horticultural chain was designed and then 7 supply chain configurations were evaluated to determine the most robust, this from the evaluation of utilities, CO2 emissions and operational performance measures such as the use of different locations. The model used for the study is a Mixed Whole Linear Programming (MIP) model that allowed to establish the configuration of the horticultural chain, opening of cross docking centers, distribution centers for vehicle evaluation to carry out the supply. The most robust configuration of the horticultural chain was established, for which, the locations that were most suitable to be enabled were defined, as well as the vehicles to be acquired for when there are low, medium and high vegetable supply levels, this involving variations in prices of sale in the different links of the chain.
\end{abstract}

Keywords: Supply chain, vegetables, horticulturist, robustness.

Cómo citar este artículo: D. Muñoz, M. Jiménez, A. Polo, K. Valencia. "Diseño robusto de la cadena de suministro hortícola: caso de estudio horticultores del municipio de Cajicá”, Revista Politécnica, vol.16, no.31 pp.41-67, 2020. DOI: 10.33571/rpolitec.v16n31a4 


\section{INTRODUCCIÓN}

La producción hortícola permite el desarrollo económico de algunas regiones endémicas de Colombia, su explotación permite la generación de empleo y el fortalecimiento de los primeros eslabones de la cadena de suministro. Para el año 2016, se presentó un incremento de $2 \%$ de áreas sembradas, pasando de 956 a 987 mil hectáreas sembradas; y se generó 527 mil empleos directos y 1.253 empleos indirectos (Departamento Administrativo Nacional de Estadísticas, 2016) que contribuyeron al desarrollo de la regiones que subsisten de este subsector de la agricultura. En Colombia existe gran diversidad en la producción hortícola, se cosechan más de 30 especies de hortalizas, entre estas la acelga, ahuyama, ají, ajo, alcachofa, apio, aromáticas, alverja, berenjena, brócoli, calabaza, cebolla cabezona, cebolla larga, cilantro, col, coliflor, espinaca, habichuela, huertos hortícolas, lechuga, pepino cohombro, pepino guiso, perejil, pimentón, remolacha, repollitas de Bruselas, repollo, tomate, zanahoria y zapallo (Senior, 2010); su producción es esencial para abastecer el mercado nacional en la capital del país y las demás ciudades y municipios que lo componen.

Cundinamarca, es un departamento líder en hectáreas cosechadas de producción hortícola (354 hectáreas) con una participación del $28 \%$ a nivel nacional (MINAGRICULTURA, 2016). Entre las hortalizas que se cosechan en este departamento, se encuentra la espinaca $(81 \%$ de siembra a nivel nacional), la lechuga (80\%), el brócoli $(72 \%)$, la alcachofa $(51 \%)$, la zanahoria $(46 \%)$ y el tomate $(12 \%$ de siembra a nivel nacional), (Senior, 2010). En la sabana de Bogotá existen 11 cultivos sobresalientes de la canasta hortícola, la mayor área cosechada es del cultivo de zanahoria con $(209,42 \mathrm{ha})$, seguido la lechuga con (189,32 ha), el cilantro con (126,56 ha), la espinaca con $(112,68 \mathrm{ha})$, el repollo con $(85,30 \%$ ha), la remolacha con $(82,35 \mathrm{ha})$, el apio con $(73,13$ ha), el coliflor con $(45,33 \mathrm{ha})$, el brócoli con $(41,93$ ha), la acelga con (35,33 ha) y el perejil con (8,25 ha) (MINAGRICULTURA, 2002). Es evidente la importancia que tiene la producción de hortalizas para el desarrollo económico del departamento.

En el municipio y áreas circunvecinas, uno de los principales motores que mueve la economía es la producción de hortalizas. Actualmente existe alrededor de 38 productores que se dedican a esta actividad, conformados entre pequeños y medianos productores. Se dedican a producir principalmente hortalizas (remolacha, zanahoria, cilantro, lechuga cebollín, coliflor, brócoli, cebolla, ajo, arveja, apio, espinaca (Secretaria De Ambiente y Desarrollo Rural, Alcaldia Municipal De Cajicá, 2018) llegando a tener volúmenes de producción de aproximadamente de 12000 toneladas al año (Bello \& Jairo, 2018). Aunque los volúmenes de producción son altos, los productores tienden a realizar la comercialización de hortalizas directamente con los intermediarios en las zonas de cultivo. Según la secretaría de ambiente y desarrollo rural del municipio de Cajicá una de las problemáticas del sector de hortalizas es la alta dependencia de intermediarios de la central de abastos que deja bajos retornos para la inversión agropecuaria (Secretaria De Ambiente y Desarrollo Rural, Alcaldia Municipal De Cajicá, 2018).

Los productores hortícolas manifiestan que, tras la negociación con los intermediarios para la venta de hortalizas no obtienen las ganancias esperadas y en algunos casos pierden dinero, debido a que, los precios ofrecidos por los intermediarios no son lo suficientemente altos como para recuperar la inversión realizada en las cosechas. Los intermediarios obtienen la mayor parte de la ganancia por abastecer los productos a las plazas de mercado; por ejemplo, el intermediario paga manojo de ajo entre $\$ 35,000$ y $\$ 40,000$ y lo vende en las plazas de mercado siempre en 10,000 pesos por encima del valor comprado, obteniendo ganancias hasta del 30 $\%$ solo por transportar y revender el producto (Bello \& Jairo, 2018). Lo mismo se presenta con productos como la lechuga (vendida por docenas) y la arveja (vendida por arrobas) que dejan ganancias a los intermediarios de más del $30 \%$ y $40 \%$ respectivamente para cada producto. De acuerdo con la información suministrada por 10 horticultores, para la producción de hortalizas se realiza una importante inversión, la cual, involucra costos de mano de obra, abono, insumos de fumigación, riegos, entre otros; Por manojo de ajo los productores invierten aproximadamente $\$ 15,000$ pesos, entre $\$ 5,000$ y $\$ 2,500$ en una docena de lechuga, y alrededor de $\$ 50,000$ y $\$ 60,000$ en una arroba de arveja. Actualmente, los horticultores solo tienen una alternativa para poder comercializar sus productos, $y$ esto es a través de los intermediarios, lo que les ha impedido el crecimiento económico.

Dado que, los productores desean adquirir una mayor ganancia por cada cosecha que realizan y no quieren depender de los intermediarios para realizar el abastecimiento de sus productos, a los productores les es difícil realizar el abastecimiento a plazas de mercado de forma independiente debido a que actualmente no existe una cadena de suministro claramente definida para ello, por lo que dependen del intermediario. Los horticultores desean abastecer 
directamente a diferentes mercados considerados estratégicos, entre estos las plazas de mercado, tiendas de barrio y fruvers de la ciudad de Bogotá. Por ello, es necesario definir la cadena de suministro para los productores hortícolas para poder realizar el abastecimiento. Sin embargo, los horticultores no saben cómo hacerlo ni qué requieren para ello.

Teniendo en cuenta que en el plan de desarrollo del municipio de Cajicá 2016-2019 "Cajicá Nuestro Compromiso" está contemplado incentivar el desarrollo y crecimiento en el territorio rural con actividades agropecuarias y el sector hortícola es la actividad agrícola más sobresaliente, según las Evaluaciones Agropecuarias de Cajicá EVAS- -del año 2013. Dicho estudio estableció que el municipio se ha caracterizado por tener una producción agropecuaria basada en cultivos transitorios de lechuga, coliflor, brócoli, espinacas, remolacha, zanahoria, maíz, en un porcentaje de $29.5 \%$, cultivos permanentes de aromáticas y frutales de durazno Pomona con un porcentaje $4.2 \%$. (Cajicá, 2016), en este sentido, estos programas serán los favorecidos a este nivel en el presente cuatrienio. Es por esto que es sumamente importante rescatar el sector agrícola en este municipio antes de que desaparezcan por completo y una manera de incentivar este tipo de actividades es fortaleciendo la comercialización de sus productos diseñando una cadena de suministro que permita llegar directamente al consumidor ampliando la cobertura de clientes, eliminando al máximo los intermediarios y así obtener una mayor utilidad de sus productos, igualmente ofreciéndole al consumidor un precio justo; además este tipo de actividades contribuye a la generación de empleo y a mejorar la calidad de vida de las personas de este municipio, igualmente motivar a nuevos agricultores a desarrollar actividades agrícolas y así otorgar a este municipio como uno de los principales abastecedores de hortalizas de la sabana cerrando un poco la brecha entre el sector urbano y rural que existe en este municipio.

Adicionalmente, mediante el fortalecimiento de la cadena de suministro y la generación asociatividad en este municipio, los agricultores podrán acceder beneficios brindados por la Alcaldía Municipal De Cajicá como lo son: i) estímulos tributarios por desarrollar actividades agropecuarias en predios con uso de suelo acorde con su vocación, recibiendo rebajas en el pago del impuesto predial, ii) el préstamo de maquinaria agrícola, dotada con todos los implementos necesarios, se ofrece a muy bajo costo y con un mínimo de requisitos para usarla, iii) Asimismo, el modelo matemático a diseñar puede ser empleado para futuras investigaciones en casos de estudios similares, al finalizar el estudio se puede identificar variables importantes a tener en cuenta para el diseño de este tipo cadenas de suministro.

El artículo está dividido en XXXXX partes, seguido a la introducción se presenta la revisión de literatura, después los materiales y métodos. Posteriormente los resultados del estudio y por ultimo las conclusiones y recomendaciones.

\section{REVISIÓN DE LITERATURA}

Ali, Maciejewski, Siegel, \& Kim (2004) definen robustez como la preservación de ciertas características deseadas del sistema, a pesar de que se presenten fluctuaciones en el comportamiento de sus partes componentes o del ambiente. La robustez se interpreta como la capacidad de establecer el resultado más estable, con baja variabilidad de las medidas clase de rendimiento como en la cadena de suministro (Lee \& Yu, 1997) y se relaciona principalmente con la superación de la vulnerabilidad de la cadena, vista como consecuencia de varias interrupciones (Tang, 2006), (Wieland \& Wallenburg, 2013). El enfoque robusto va dirigido a cadenas de suministro eficientes, ya que tiene como objetivo reconocer y explorar la incertidumbre que es inherente a las cadenas y a partir de eso tomar decisiones de planificación que producirán resultados más predecibles y estables (Van \& Vanmaele, 2002). Es necesario que las cadenas de suministro se diseñen de manera robusta con respecto a condiciones inciertas de operación para que las ineficiencias operativas como retrasos e interrupciones no afecten el sistema (Santoso, Ahmed, Goetschalckx, \& Shapiro, 2005). La robustez de la cadena de suministro puede ser vista como el grado en que esta muestra un rendimiento aceptable en sus indicadores clave de rendimiento (KPI) durante y después de un evento inesperado que causó disturbios en uno o más procesos logísticos (Vlajic, Van der Vorst, \& Hendrix, 2009).

Precisamente Ali et al. (2004) proponen un Procedimiento denominado FePIA, este es usado a manera de métrica de robustez, es decir, con él se determina cuantitativamente qué tan robusto es un sistema. El presente artículo está basado esencialmente en el uso de la metodología propuesta por Tordecilla-Madera, \& González-Rodríguez (2012) y en el de Tordecilla et al. (2016), los cuales están basados en el trabajo de Ali et al. (2004). En el trabajo de Tordecilla et al. (2016) se realizó un diseño robusto para el sistema logístico de almacenamiento y refrigeración de leche de una cooperativa láctea colombiana utilizando programación binaria. Se 
adaptó este proceso para aplicarlo en la cadena hortícola. Adicional, se involucró el trabajo de Vianchá (2014), quien presenta un marco de referencia en los modelos y configuraciones de

\section{MATERIALES Y MÉTODOS}

Para el diseño de una cadena de suministro hortícola robusta, se planteó un procedimiento basado en dos etapas. i) la construcción de modelo matemático que permite el diseño y estudio de la cadena de suministro, y ii) el establecimiento de la metodología para evaluar la robustez de la cadena de suministro. En cuanto a la primera etapa, se planteó y solucionó un modelo matemático en GAMS 22.5, con un cadenas de suministro agrícolas, específicamente en productos perecederos. Así pues, logró dar un aporte al conocimiento con la adaptación del Procedimiento FePIA al contexto de la cadena hortícola. procesador Intel Core i5 de octava generación. E modelo permitió la representación de una cadena de suministro Hortícola ubicada en el departamento de Cundinamarca - Colombia, específicamente en el municipio de Cajicá. En la Fig. 1 se visualiza la cadena de suministro que permite representar el modelo matemático planteado:

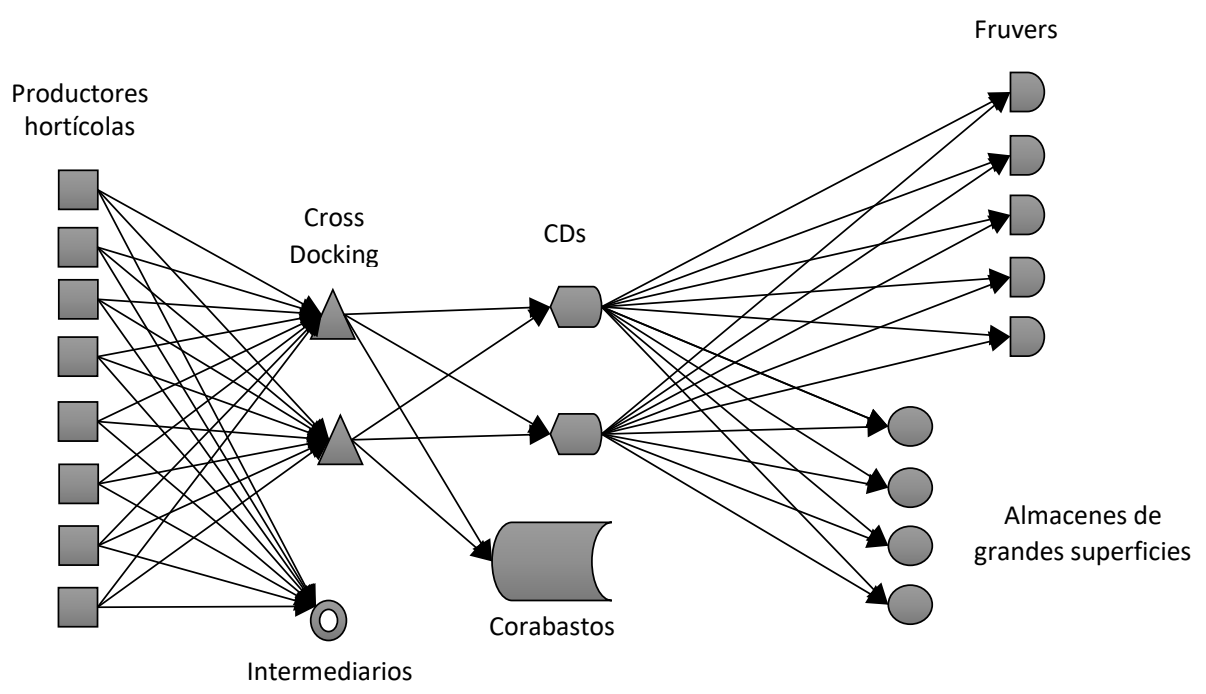

Fig. 1. Grafo representativo de la cadena de suministro hortícola planteada

Es importante resaltar que el modelo matemático diseñado, es planteado con el propósito de determinar la posibilidad de que el productor agrícola no dependa directamente del intermediario, sino que, pueda expandir su mercado a otros puntos de demanda como fruves, almacenes de grandes superficies y Corabastos. Por tanto, el modelo
$i$ : Producto (hortaliza)
$j:$ productor (horticultor)
$k$ : Puntos de cross docking
l: Centros de distribuciòn $m$ : Fruvers
$n$ : Almacenes de grandes superficies

$$
\begin{aligned}
& k=1,2 \ldots K \\
& l=1,2 \ldots L \\
& m=1,2 \ldots M
\end{aligned}
$$$$
i=1,2 \ldots I
$$$$
j=1,2 \ldots J
$$$$
l=1,2 \ldots L
$$$$
n=1,2 \ldots N
$$

permite determinar lo nodos de la cadena a los que conviene entregar la producción y a su vez determinar los requerimientos de infraestrura y transportes necesarios para ello. El modelo consta de doce (12) tipos de conjuntos, estos se describen a continuación: vo: Vehiculos para el trans. entre productores y cross docking

vp: Vehiculos para el trans. entre puntos de cross doking y CDs

vq:Vehiculos para el trans. entre CDs y fruvers

vr: Vehiculos para el trans. entre CDs y almacenes de grandes sup.

vt: Vehiculos para el trans. entre puntos de cross docking y corabastos

$t$ : Periodo de análisis (Mensual)

$$
\begin{aligned}
& v o=1,2 \ldots V O \\
& v p=1,2 \ldots V P \\
& v q=1,2 \ldots V Q \\
& v r=1,2 \ldots V R \\
& v t=1,2 \ldots V T
\end{aligned}
$$


La información paramétrica del modelo reúne datos asociados a la ubicación de cada uno de los nodos, distancias entre nodos, capacidades de transporte, almacenamiento, niveles de oferta demanda e información relacionadas con los costos de operación. Los parámetros se presentan a continuación:

$A J_{j}:$ Absica del horticultor $j$

$O J_{j}$ : Ordenada del horticultor $j$

$A K_{k}:$ Abscisa del punto de cross docking $k$

$O K_{k}$ : Ordenda del punto de cross docking $k$

$A L_{l}$ : Abscisa del almacen de distribución l

$O L_{l}$ : Ordenada del almacen de distribución $l$

$A M_{m}:$ Abscisa del fruver $m$

$O M_{m}:$ Ordena del fruver $m$

$A N_{n}:$ Abscisa de gran superficie $n$

$O N_{n}$ : Ordenada de gran superficie $n$

Para el cálculo de la distancia entre los diferentes eslabones de la cadena se hace de forma lineal, esto empleando la ecuación para distancias euclidianas. Es importante en el cálculo de distancia multiplicar por $110.6 \mathrm{~km}$ para hacer la conversión de grados a kilómetros, dado que la información de la ubicación de cada uno de los nodos de la cadena se recolecta a partir del uso de GPS. Esto se muestra a continuación:

$D J K_{j k}=$ Distancia entre productores $j$ y puntos de cross docking $k$

$$
D J K_{j k}=110.6 * \sqrt{\left(A J_{j}-A K_{k}\right)^{2}+\left(O J_{j}-O K_{k}\right)^{2}}
$$

$D K L_{k l}$ : Distancia entre los puntos de cross docking $k$ y centros de distribución $l$

$$
D K L_{k l}=110.6 \sqrt{\left(A K_{k}-A L_{l}\right)^{2}+\left(O K_{k}-O L_{l}\right)^{2}}
$$

$D L M_{l m}$ : Distancia entre los centros de distribuciòn $l$ y los fruvers $m$

$$
D L M_{l m}=110.6 \sqrt{110.6\left(A L_{l}-A M_{m}\right)^{2}+\left(O L_{l}-O M_{m}\right)^{2}}
$$

$D L N_{l n}$ : Distancia entre los puntos de cross docking l y almacenes de cadena $n$

$$
D L N_{l n}=110.6 \sqrt{\left(A L_{k}-A N_{n}\right)^{2}+\left(O L_{l}-O N_{n}\right)^{2}}
$$

$D K C_{k}$ : Distancia entre los puntos de cross docking y corabastos

$$
D K C_{k}=\sqrt{\left(A K_{k}-A C O\right)^{2}+\left(O K_{k}-O C O\right)^{2}}
$$

$O F_{i j t}:$ Oferta de hortaliza $i$ del productor $j$ en el periodo $t$.

$C C R_{k}$ : Capacidad de recepción del punto de cross docking $k(\mathrm{~kg})$

$C C D_{l}$ : Capacidad de almacenamiento del centro de distribución l $(\mathrm{kg})$

$D I_{i t}$ : Demanda de hortalizas $i$ en el periodo t por parte de los intermediarios $(\mathrm{kg})$

$D M_{\text {imt }}$ : Demanda de hortalizas i en los fruvers $m$ en el periodo $t(\mathrm{~kg})$

$D N_{\text {int }}$ : Demanda de hortalizas $i$ en los almacenes de grandes superficies $n$ en el periodo $t(\mathrm{~kg})$

$D C_{i t}$ : Demanda de hortalizas $i$ en corabastos en el periodo $t(\mathrm{~kg})$

$P_{P I}$ : Precio medio de venta de un $\mathrm{kg}$ de hortaliza $i$ a los intermediarios

$P M M_{i}$ : Precio medio de venta de un $\mathrm{kg}$ de hortaliza $i$ en el fruver $m$

$P N M_{i}$ : Precio medio de venta de un $\mathrm{kg}$ de hortaliza $i$ en almacenes $n$

$P C M_{i}$ : Precio medio de venta de un $\mathrm{kg}$ de hortaliza i en corabastos

$K P_{i t}:$ Costo medio de producción de la hortaliza $i$ (\$ $\left.-\mathrm{kg}\right)$

$K C R_{k}$ : Costo de operación (arrendamiento y servicios)del punto de cross docking $k$

$K C D_{l}$ : Costo de operación (arrendamiento y servicios) del centro de distribución l

El cálculo de los costos de transporte entre los diferentes nodos de la cadena, se determinó a partir del consumo de combustible por kilogramo por kilómetro de los diferentes vehículos que se cotizaron para el estudio. Para ello se emplean los siguientes parámetros:

$\mathrm{TO}_{v o}=$ costo por kilometro de vehiculo vo

$C T 1_{j, k, v o}$ : Costo de trans. por $\mathrm{kg}$ desde el horti. $j$ hasta el cross docking $k$ el vehiculo vo 
$C T 1_{j, k, v o}=T O_{v o} * D J K_{J k}$

$T P_{v p}=$ costo por kilometro de vehiculo $v p$

$C T 2_{k, l, v p}$ : Costo de trans. por $\mathrm{kg}$ desde el cross docking $k$ hasta el CD l en el vehiculo vp.

$C T 2_{k, l, v p}=T P_{v p} * D K L_{k l}$

$T Q_{v q}=$ costo por kilometro de vehiculo $v q$

$C T 3_{l, m, v q}:$ Costo de trans. por $\mathrm{kg}$ desde el CD l hasta el fruver $m$ en el vehiculo vq $C T 3_{l, m, v q}=T Q_{v q} * D L M_{l m}$

$T R_{v r}=$ Costo por kilometro de vehiculo vr

$C T 4_{l, n, v r}$ : Costo de trans.por $\mathrm{kg}$ desde el CDs l hasta el almacene $n$ en el vehiculo vr $C T 4_{l, n, v r}=T R_{v r} * D L N_{l n}$

$T T_{v t}=$ Costo por kilometro de vehiculo vt

$C T 5_{k, v t}$ : Costo de trans. por $k g$ desde cross docking $k$ hasta corabastos $C T 5_{k, v t}=T T_{v t} * D K C_{k}$

Las capacidades de transporte de los vehículos fueron determinadas a partir del máximo que pueden cargar los vehículos en un viaje, y multiplicándola por el número de viajes estimados que se pueden realizar en un mes, esto con el propósito de calcular la capacidad mensual de transporte de cada uno de los vehículos.

$K T 1_{v o}:$ Capacidad de transporte de vehiculos tipo vo $K T 2_{v p}$ : Capacidad de transporte de vehiculos tipo vp $K T 3_{v q}$ : Capacidad de transporte de vehiculos tipo vq $K T 4_{v r}:$ Capacidad de transporte de vehiculos tipo vr $K T 5_{v t}$ : Capacidad de transporte de vehiculos tipo vt $I C R_{k}:$ Inversión inicial necesaria para la operación del punto de cross docking ICD $D_{l}$ Inversión inicial necesaria para la operación del centro de distribución $I N 1_{v o}$ : Inversión inicial para la adquisición de vehiculos tipo vo $I N 2_{v p}$ : Inversión inicial para la adquisición de vehiculos tipo tipo vp

$I N 3_{v q}$ : Inversión inicial para la adquisición de vehiculos tipo vq

$I N 4_{v r}$ : Inversión inicial para la adquisición de vehiculos tipo vr

IN $5_{v t}:$ Inversión inicial para la adquisición de vehiculos tipo vt

$D P 1_{\text {vot }}$ : Costo de depreciación del medio de transporte vo (lineal 120 meses -10 años) $D P 1_{\text {vot }}=I N 1_{\text {vo }} /(120)$

$D P 2_{\text {vpt }}$ : Costo de depreciación del medio de transporte vp (lineal 120 meses -10 años) $D P 2_{v p t}=I N 2_{v p} /(120)$

$D P 3_{\text {vqt }}$ : Costo de depreciación del medio de transporte vq(lineal 120 meses -10 años) $D P 3_{v q t}=I N 3_{v q} /(120)$

$D P 4_{\text {vrt }}$ : Costo de depreciación del medio de transporte vr (lineal 120 meses - 10 años) $D P 4_{v r t}=I N 4_{v r} /(120)$

$D P 5_{v t t}$ : Costo de depreciación del medio de transporte vt (lineal 120 meses -10 años) $D P 5_{v t t}=I N 5_{v t} /(120)$

$\mathrm{CO}_{v_{0}}$ : Coeficientes de emision de CO2 para los vehiculos vo (gramos $\left.\mathrm{kg}-\mathrm{km}\right)$

$\mathrm{CO} 2_{v p}$ : Coeficientes de emision de CO2 para los vehiculos vp (gramos $\mathrm{kg}-\mathrm{km}$ )

$\mathrm{CO}_{v q}$ : Coeficientes de emision de CO2 para los vehiculos vq (gramos $\mathrm{kg}-\mathrm{km}$ )

$\mathrm{CO}_{\text {vr }}$ : Coeficientes de emision de C02 para los vehiculos vr (gramos $\mathrm{kg}-\mathrm{km}$ )

$\mathrm{CO5}_{v t}$ : Coeficientes de emision de CO2 para los vehiculos vt (gramos $\mathrm{kg}-\mathrm{km}$ )

Se establecieron treinta (30) tipos de variables para ver el comportamiento de la cadena de suministro, estas son:

FO: Función objetivo (utilidad)

$I N G$ : Ingreso por ventas

CTO: Costos totales de operación

CTR $1_{\text {vot }}$ : Costos de trans. desde horticultores hasta cross docking

$C T R 2_{\text {vpt }}$ : Costos de trans. desde cross docking a CDs 
$C T R 3_{\text {vqt }}$ : Costos de trans. desde CDs a fruvers

$C T R 4_{\text {vrt }}$ : Costos de trans. desde CDs a alamcenes de grandes superficies

CTR $5_{v t t}$ : Costos de trans. desde cross docking hasta corabastos

$C T C D_{t}$ : Costo del salario de los conductores de los vehiculos adquiridos en el periodo $t$

$C D P V 1_{v o}$ : Costos de depreciación de los vehiculos tipo vo

$C D P V 2_{v p}$ : Costos de depreciación de los vehiculos tipo vp

$C D P V 3_{v q}$ : Costos de depreciación de los vehiculos tipo vq

$C D P V 4_{v r}$ : Costos de depreciación de los vehiculos tipo vr

$C D P V 5_{v t}$ : Costos de depreciación de los vehiculos tipo vt

COP $1_{k t}$ : Costos de operación de puntos de croos docking

COP $2_{l t}$ : Costos de operación de puntos centros de distribución

$X O_{i, j, k, v o, t}$ : Cantidad de hortaliza $i$ a enviar desde $j$ hasta $k$ en el vehiculo vo en el periodo $t(k g)$

$X P_{\text {iklvpt }}$ : Cantidad de hortaliza $i$ a enviar $k$ hasta l en el vehiculo vp en el periodo $t(\mathrm{~kg})$

$X Q_{\text {ilmvqt }}$ : Cantidad de hortaliza $i$ a enviar l hasta $m$ en vehiculo vq en el periodo $t(\mathrm{~kg})$

$X R_{\text {ilnvrt }}$ : Cantidad de hortaliza $i$ a enviar l hasta $n$ en el vehiculo vr en el periodo $t(\mathrm{~kg})$

$X T_{i k v t t}$ : Cantidad de hort. $i$ a enviar desde $k$ hasta corabastos en el vehiculo vt en el periodo $t(\mathrm{~kg})$

$X U_{i j t}$ : Cantidad de hortaliza $i$ que cada horticultor debe vender a intermediarios en el periodo $t(\mathrm{~kg})$

ECO: Cantidad de emisiones CO2 emitidas (gramos)

$B O_{v o}:$ Cantidad de vehiculos tipo vo que se deben comprar

$B P_{v p}:$ Cantidad de vehiculos tipo vp que se deben comprar

$B Q_{v q}$ : Cantidad de vehiculos tipo vq que se deben comprar

$B R_{v r}$ : Cantidad de vehiculos tipo vr que se deben comprar

$B T_{v t}:$ Cantidad de vehiculos tipo vt que se deben comprar

$A 1_{k}$ : Binario si se debe abrir o no la zona de Cross docking $k$

$A 2_{l}$ : Binario se se debe abrir o no el centro de distribución l

El modelo está sujeto a las siguientes ecuaciones:

$$
\text { Max } \quad F O=I N C-C T O
$$

La ecuación (1) indica la función objetivo la cual busca maximizar las utilidades de los agricultores.

$$
\begin{aligned}
I N G=\sum_{i}^{I} \sum_{l}^{L} \sum_{m}^{M} \sum_{v q}^{V Q} \sum_{t}^{T}\left(X Q_{i, l, m, v q, t} * P M M_{i}\right)+\sum_{i}^{I} \sum_{l}^{L} \sum_{n}^{N} \sum_{v r}^{V R} \sum_{t}^{T}\left(X R_{i, l, n, v r, t} * P N M_{i}\right) \\
+\sum_{i}^{I} \sum_{k}^{K} \sum_{v t}^{V T} \sum_{t}^{T}\left(X T_{i, k, v t, t} * P C M_{i}\right)+\sum_{i} \sum_{j}^{J} \sum_{t}^{T}\left(X U_{i, j, t} * P I M_{i}\right)
\end{aligned}
$$

La ecuación (2) indica los ingresos los cuales son calculados a partir de la suma de las cantidades enviadas a cada cliente multiplicado por su correspondiente precio de venta. 


$$
\begin{aligned}
C T O=\sum_{v o}^{V O} \sum_{t}^{T}( & \left.C R 1_{v o, t}\right)+\sum_{v p}^{V P} \sum_{t}^{T}\left(C T R 2_{v p, t}\right)+\sum_{v q}^{V Q} \sum_{t}^{T}\left(C T R 3_{v q, t}\right) \\
& +\sum_{v r}^{V R} \sum_{t}^{T}\left(C T R 4_{v r, t}\right)+\sum_{v t}^{V T} \sum_{t}^{T}\left(C R T 5_{v t, t}\right)+\sum_{v o}^{V O}\left(C D P V 1_{v o}\right) \\
& +\sum_{v p}^{V P}\left(C D P V 2_{v p}\right)+\sum_{v q}^{V Q}\left(C D P V 3_{v q}\right)+\sum_{v r}^{V R}\left(C D P V 4_{v r}\right) \\
& +\sum_{v t}^{V T}\left(C D P V 5_{v t}\right)+\sum_{k}^{K} \sum_{t}^{T}\left(C O P 1_{k, t}\right)+\sum_{l}^{T} \sum_{t}^{T}\left(C O P 2_{l, t}\right) \\
& +\sum_{i}^{I} \sum_{j}^{J} \sum_{k}^{K} \sum_{v o}^{V O} \sum_{t}^{T}\left(K P_{i, t} * X O_{i, j, k, v o, t}\right) \\
& +\sum_{i}^{I} \sum_{j}^{J} \sum_{t}^{T}\left(K P_{i, t} * X U_{i, j, t}\right)+\sum_{t}^{T}\left(C T C D_{t}\right)
\end{aligned}
$$

Con la ecuación (3) se puede calcular el costo total de operación, los cuales incluyen los costos de transporte, costos de depreciación, costos de operación en los diferentes nodos que articulan la cadena de suministro.

$$
\begin{aligned}
C T R 1_{v o t} & =\sum_{i}^{I} \sum_{j}^{J} \sum_{k}^{K} X O_{i, j, k, v o, t} * C T 1_{j, k, v o} \forall v o, t \\
C T R 2_{v p t} & =\sum_{i}^{I} \sum_{k}^{K} \sum_{l}^{L} X P_{i, k, l v v p, t} * C T 2_{k, l, v p} \forall v p, t \\
C T R 3_{v q t} & =\sum_{i}^{I} \sum_{l}^{L} \sum_{m}^{M} X Q_{i, l, m, v q, t} * C T 3_{l, m, v q} \forall v q, t \\
C T R 4_{v r t} & =\sum_{i}^{I} \sum_{l}^{L} \sum_{n}^{N} X R_{i, l, n, v r, t} * C T 4_{l, n, v r} \forall v r, t \\
C T R 5_{v t t} & =\sum_{i}^{I} \sum_{k}^{K} X T_{i, k, v t, t} * C T 5_{k, v t} \forall v t, t
\end{aligned}
$$

A partir de las ecuaciones $(4,5,6,7,8)$ se puede calcular el costo de transporte de las hortalizas, entre los diferentes eslabones de acuerdo con el tipo de vehículo que se emplee y a los volúmenes de producto que transportar.

$$
C T C D_{t}=S *\left[\sum_{v o}^{V O} B O_{v o}+\sum_{v p}^{V P} B P_{v p}+\sum_{v q}^{V Q} B Q_{v q}+\sum_{v r}^{V R} B R_{v r}+\sum_{v t}^{V T} B T_{v t}\right]
$$

La ecuación (9) permite calcular el costo asociado al salario que se debe pagar a los conductores que se requieran para el transporte de mercancía entre los diferentes eslabones de la cadena, esto teniendo en cuenta el total de vehículos que se deben comprar para el transporte.

$$
\begin{aligned}
& C D P V 1_{v o}=\sum_{t}^{T} B O_{v o} * D P 1_{v o, t} \\
& C D P V 2_{v p}=\sum_{t}^{T} B P_{v p} * D P 2_{v p, t} \\
& C D P V 3_{v q}=\sum_{t}^{T} B Q_{v q} * D P 3_{v q, t}
\end{aligned}
$$




$$
\begin{aligned}
& C D P V 4_{v r}=\sum_{t}^{T} B R_{v r} * D P 4_{v r, t} \\
& C D P V 5_{v t}=\sum_{t}^{T} B T_{v t} * D P 5_{v t, t}
\end{aligned}
$$

Con las ecuaciones $(10,11,12,12,13,14)$ se puede calcular los costos de depreciación de los vehículos necesarios para realizar el abastecimiento de hortalizas, esto dependiendo de la cantidad de vehículos que se requiera adquirir para el transporte de mercancía entre los diferentes nodos.

$$
\begin{gathered}
C O P 1_{k t}=A 1_{k} * K C R_{k} \quad \forall k, t \\
C O P 2_{l t}=A 2_{l} * K C D_{l} \quad \forall k,
\end{gathered}
$$

Con las ecuaciones $(15,16)$ se pueden calcular los costos asociados a la operación de los puntos de Cross docking y los centros de distribución que se requieran abrir para el funcionamiento de la cadena.

$$
\sum_{k}^{K} \sum_{v o}^{V O}\left(X O_{i, j, k, v o, t}\right) \leq O F_{i j t} \quad \forall i, j, t
$$

La ecuación (17) permite restringir la oferta de hortalizas que disponen los productores en los diferentes periodos de evaluación.

$$
\begin{gathered}
\sum_{l}^{l} \sum_{v q}^{V Q}\left(X Q_{i, l, m, v q, t}\right) \leq D M_{i m t} \forall i, m, t \\
\sum_{i}^{I} \sum_{v r}^{V R}\left(X R_{i, l, n, v v, t}\right) \leq D N_{i n t} \forall i, n, t \\
\sum_{k}^{K} \sum_{v t}^{V T}\left(X T_{i, k, v t, t}\right) \leq D C_{i t} \forall i, t \\
\sum_{j}^{J}\left(X U_{i j t}\right) \leq D T_{i t} \forall i, t
\end{gathered}
$$

Las ecuaciones $(18,19.20$ y 21$)$ permiten restringir las cantidades que se envían de acuerdo con las demandas existentes en los diferentes nodos. Específicamente la demanda los fruvers, almacenes de cadena, Corabastos e intermediarios.

$$
\begin{gathered}
\sum_{i}^{I} \sum_{j}^{J} \sum_{v o}^{V O}\left(X O_{i, j, k, v o, t}\right) \leq C C R_{k} * A 1_{k} \forall i, t \\
\sum_{i}^{I} \sum_{k}^{K} \sum_{v p}^{V P}\left(X P_{i, k, l v p, t}\right) \leq C C D_{l} * A 2_{l} \forall l, t
\end{gathered}
$$

Las ecuaciones (22 y 23) permiten limitar los flujos de productos a través de los centros de distribución y Cross docking acorde con su capacidad máxima.

$$
\begin{aligned}
& \sum_{i}^{I} \sum_{j}^{J} \sum_{k}^{K}\left(X O_{i, j, k, v o, t}\right) \leq K T 1_{v o} * B O_{v o} \forall v o, t \\
& \sum_{i}^{I} \sum_{k}^{K} \sum_{l}^{L}\left(X P_{i, k, l, v p, t}\right) \leq K T 2_{v p} * B P_{v p} \forall v p, t
\end{aligned}
$$




$$
\begin{gathered}
\sum_{i}^{I} \sum_{l}^{L} \sum_{m}^{M}\left(X Q_{i, l, m, v q, t}\right) \leq K T 3_{v q} * B Q_{v q} \forall v q, t \\
\sum_{i}^{I} \sum_{l}^{L} \sum_{n}^{N}\left(X R_{i, l, n, v r, t}\right) \leq K T 4_{v r} * B R_{v r} \forall v r, t \\
\sum_{i}^{I} \sum_{k}^{K}\left(X T_{i, k, v t, t}\right) \leq K T 5_{v t} * B T_{v t} \forall v t, t
\end{gathered}
$$

Las ecuaciones $(24,25,26,27$ y 28$)$ permiten restringir la capacidad máxima que se puede transportar entre los diferentes eslabones para los vehículos de acuerdo con la cantidad de vehículos que se adquieran para el abastecimiento.

$$
\begin{gathered}
\sum_{j}^{J} \sum_{v o}^{V O}\left(X O_{i, j, k, v o, t}\right)=\sum_{l}^{L} \sum_{v p}^{V P}\left(X P_{i, k, l, v p, t}\right)+\sum_{v t}^{V T}\left(X T_{i, k, v t, t}\right) \forall i, k, t \\
\sum_{k}^{K} \sum_{v p}^{V P}\left(X P_{i, k, l, v p, t}\right)=\sum_{m}^{M} \sum_{v q}^{V Q}\left(X Q_{i, l, m, v q, t}\right)+\sum_{n}^{N} \sum_{v r}^{V R}\left(X R_{i, l, n, v r, t}\right) \forall i, l, t
\end{gathered}
$$

Permiten realizar el equilibro en los nodos intermedios de la cadena específicamente en los puntos Cross docking (Ecuación 30) y centros de distribución (Ecuación 31).

$$
\begin{aligned}
& E C O=\sum_{i}^{I} \sum_{j}^{J} \sum_{k}^{K} \sum_{v o}^{V O} \sum_{t}^{T}\left(C O 1_{v o} * X O_{i, j, k, v o, t} * D J K_{j k}+\sum_{i}^{I} \sum_{k}^{K} \sum_{l}^{L} \sum_{v p}^{V P} \sum_{t}^{T} C O 2_{v p} * X P_{i, k, l, v p, t}\right. \\
& * D K L_{k l}+\sum_{i}^{I} \sum_{l}^{L} \sum_{m}^{M} \sum_{v q}^{V Q} \sum_{t}^{T}\left(C O 3_{v q} * X Q_{i, l, m, v q, t}\right)+D L M_{l m} \\
&+\sum_{i}^{I} \sum_{l}^{L} \sum_{n}^{N} \sum_{v r}^{V R} \sum_{t}^{T}\left(C O 4_{v r} * X R_{i, l, n, v r, t}\right) * D L N_{l n}+\sum_{i}^{I} \sum_{k}^{K} \sum_{v t}^{V T} \sum_{t}^{T}\left(C O 5_{v t}\right. \\
&\left.* X T_{i, k, v t, t}\right)+D K C_{k}
\end{aligned}
$$

A partir de la ecuación (32) se realiza el cálculo de las emisiones de Co2, esto de acuerdo con los volúmenes de producto a transportar y la distancia recorrida durante el transporte entre los diferentes nodos.

$$
\begin{gathered}
F O \in \mathcal{R} \\
N G, C T O, C T R 1_{v o, t}, C T R 2_{v p, t}, C T R 3_{v q, t}, C T R 4_{v r, t}, C T R 5_{v t t}, C T C D_{t}, C D P V 1_{v o}, \\
C D P V 2_{v p}, C D P V 3_{v q}, C D P V 4_{v r}, C D P V 5_{v t}, C O P 1_{k t}, C O P 2_{l t}, X O_{i j k v o t}, X P_{i k l v p t}, \\
X Q_{i l m v q t}, X R_{i l n v r t}, X T_{i k v t t}, X U_{i j t}, E C O \geq 0 \\
B 0_{v o}, B P_{v p}, B Q_{v q}, B R_{v r}, B T_{v t} \in \mathbb{Z} \\
A 1_{k}, A 2_{l}\left\{\begin{array}{l}
0 \\
1
\end{array}\right.
\end{gathered}
$$

Las ecuaciones $(33,34$ y 35) son restricción de pertenecía a numero reales, enteros y binarios.

En cuanto a la segunda etapa, la metodología de análisis de robustez se construyó a partir del trabajo de (Tordecilla, Polo, Muñoz, \& González, 2016), esta consta de siete (7) fases que permiten la identificación de la cadena de suministro que se ha más robusta a partir del análisis de diferentes indicadores que se describen en la Fig. 2. 


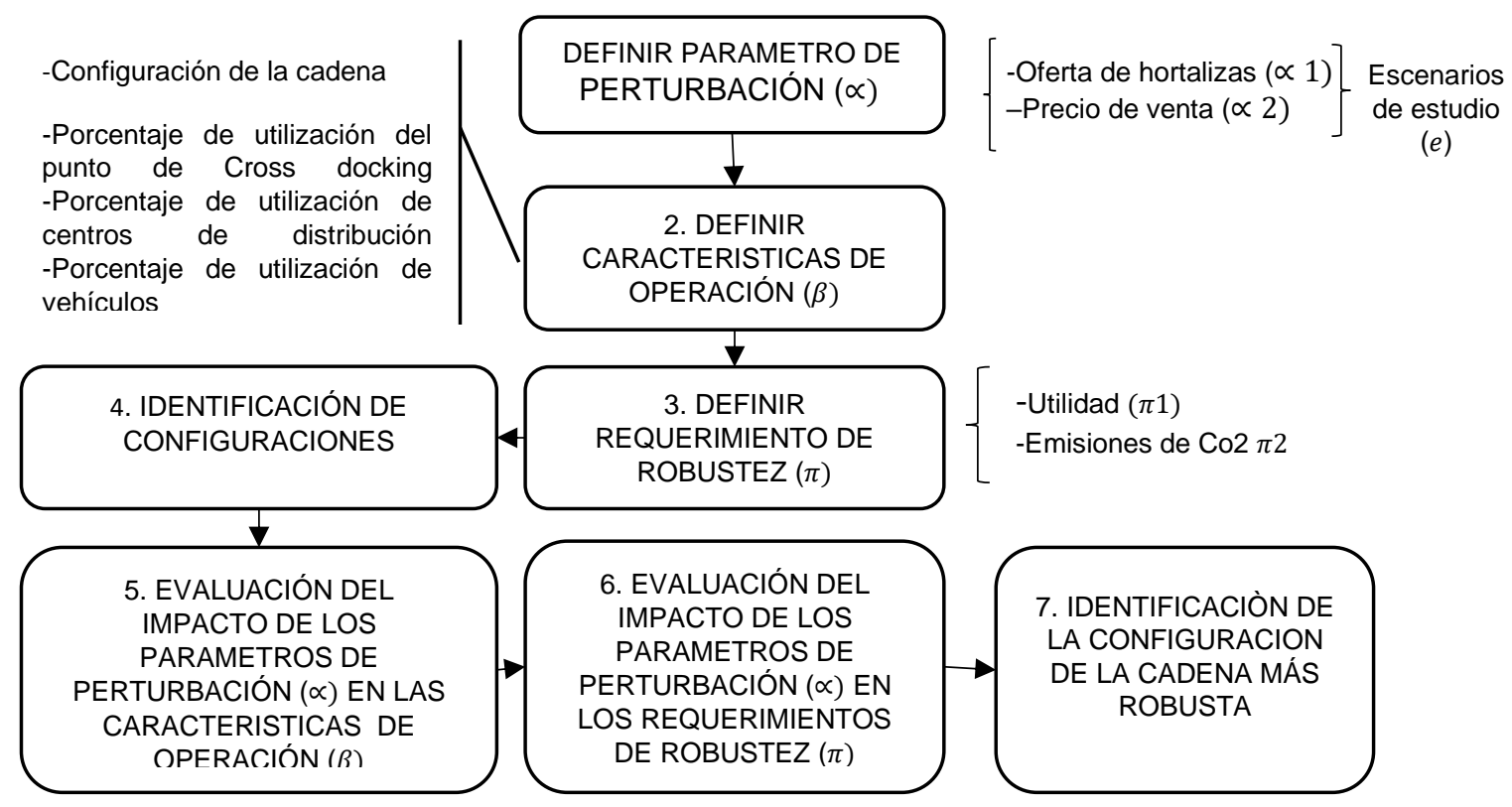

Fig. 2. Metodología empleada para el análisis de robustez adaptada del trabajo de (Tordecilla, Polo, Muñoz, \& González, 2016)

A partir de los parámetros de perturbación, permiten definir los escenarios de estudio. Esto variando el porcentaje de oferta que pueden dar los productores hortícolas y el precio de venta de la hortaliza, los cuales en el mercado son inversamente proporcionales. A mayor oferta de hortaliza, menor es el precio de venta de esta y viceversa en cuanto menor sea la oferta, los productores pueden vender la producción a mayor precio. Es necesario establecer un volumen máximo y mínimo de producción de hortaliza, de igual forma un máximo y un mínimo en el precio de venta. Se tienen en cuenta los siguientes parámetros:

$P I L_{i}:$ Precio minimo de venta de un $\mathrm{kg}$ de hortaliza $i$ a los intermediarios

$P M L_{i}$ : Precio minimo de venta de un $\mathrm{kg}$ de hortaliza $i$ en los fruvers

$P N L_{i}:$ Precio minimo de venta de un $\mathrm{kg}$ de hortaliza $i$ en los almacenes de grandes superficies

$P C L_{i}:$ Precio minimo de venta de un $\mathrm{kg}$ de hortaliza $i$ en corabastos

$P I U_{i}:$ Precio máximo de venta de un $\mathrm{kg}$ de hortaliza $i$ a los intermediarios

$P M U_{i}=$ Precio máximo de venta de un $\mathrm{kg}$ de hortaliza $i$ en los fruvers

$P N U_{i}$ : Precio máximo de venta de un $\mathrm{kg}$ de hortaliza $i$ en los almacenes de grandes superficies

$P C U_{i}$ : Precio máximo de venta de un $\mathrm{kg}$ de hortaliza $i$ en corabastos

Con estos nuevos parámetros e posible determinar el precio medio de venta bajo el cual se debe realizar la corrida del modelo. El nuevo precio de venta de las hortalizas en los diferentes eslabones se calcula así:

$P I M_{i}:$ Precio medio de venta de un $\mathrm{kg}$ de hortaliza $i$ a los intermediarios

$$
\text { PIM }_{i}=P I L_{i}+\Delta \mathrm{P} *\left(P I U_{i}-P I L_{i}\right)=\propto 2
$$

$P M M_{i}$ : Precio medio de venta de un $\mathrm{kg}$ de hortaliza $i$ en el fruver $m$

$$
P M M_{i}=P M L_{i}+\triangle \mathrm{P} *\left(P M U_{i}-P M L_{i}\right)
$$

$P N M_{i}$ : Precio medio de venta de un $\mathrm{kg}$ de hortaliza $i$ en almacenes $n$

$$
P N M_{i}=P N L_{i}+\triangle \mathrm{P} *\left(P N U_{i}-P N L_{i}\right)
$$

$P C M_{i}:$ Precio medio de venta de un $\mathrm{kg}$ de hortaliza $i$ en corabastos

$$
P C M_{i}=P C L_{i}+\triangle \mathrm{P} *\left(P C U_{i}-P C L_{i}\right)
$$


Se establece el parámetro $\Delta \mathrm{P}$ para calcular una variación especifica entre el precio mínimo y el precio máximo de venta, esto de acuerdo al escenario de estudio deseado. Algo similar ocurre con los volúmenes de producción de hortalizas. La variación que se realiza a la oferta de hortalizas se hace con el parámetro $(\Delta 0)$ que indica el porcentaje de hortaliza que se pude producir en un escenario deseado. Se calcula el parámetro $O F_{i j t}$ de la siguiente forma:

$O F_{i j t}:$ Oferta calculada de acuerdo al escenarios establecido.

Donde:

$$
O F_{i j t}=\text { OFERTA } A_{i j t} * \Delta \mathrm{O}=\propto 1
$$

OFERT $A_{i j t}$ : Oferta máxima de hortalizas $i$ del producto $j$ en el periodo $t(\mathrm{~kg})$

Una ver entendidos el modelo matemático y la metodología de robustez planteada, se procede a explicar los resultados obtenidos.

\section{RESULTADOS DEL ESTUDIO}

El modelo y la metodología se aplicaron con un conjunto de productores del municipio de Cajicá Colombia, que manifestaron interés establecer alternativas para la comercialización de hortalizas. En total se contó con la participación de 19 productores que se dedican a la explotación 14 tipos de hortalizas (lechuga, brócoli coliflor, cilantro, espinaca, zanahoria, remolacha, arveja, papa, perejil, apio, acelga, calabacín y ajo). Se realizó el estudio específicamente con cinco tipos de hortalizas que son las de mayor producción y las que mayor expectativa de retribución puede dejar para los productores involucrados en el estudio. Hortaliza es producida y comercializada principalmente a intermediarios de la cadena y a Corabastos. En la Fig. 3 se puede ver la estructura actual de la cadena de suministro de los productores involucrados en el estudio.

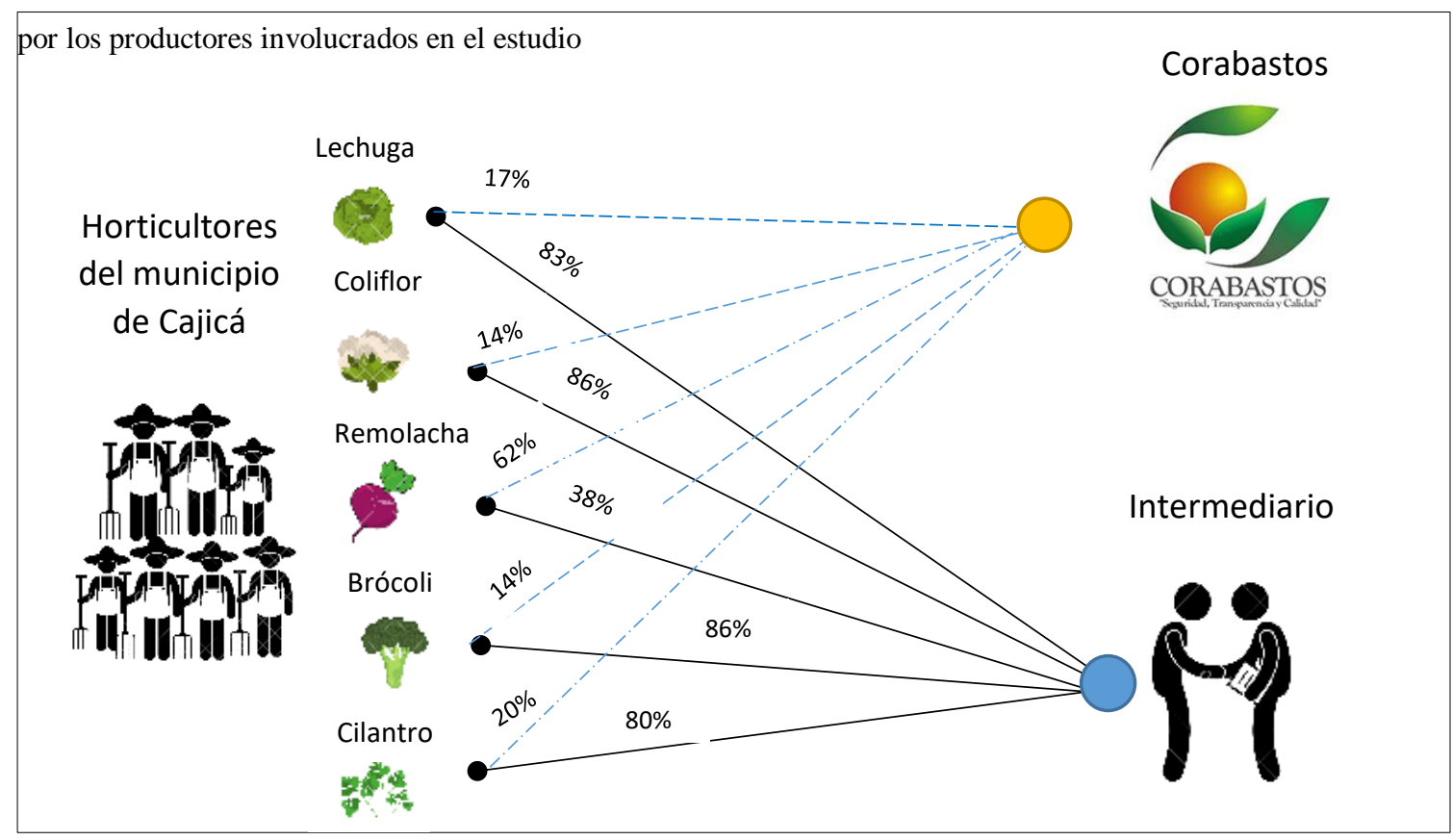

Fig. 3.Cadena actual de suministro de los productos estudiados

Evidentemente, los horticultores no solo tienen contacto directo con los intermediarios y Corabastos: sin embargo, se desea abastecer otros eslabones de la cadena teniendo en cuenta las limitaciones de oferta de los productores, dado que actualmente se tiene dificultades con la comercialización y de hortalizas, reciben muy baja retribución económica cuando logran volúmenes altos de producción. Se decidió evaluar la cadena hortícola para establecer los eslabones a los que deben abastecer los 19 
productores involucrando temporadas bajas y altas de producción, así como el precios mínimos y máximos de venta de la lechuga, coliflor, remolacha,

\section{Definición de los parámetros de perturbación}

Como se había establecido en la metodología, se definieron dos (2) parámetros de perturbación, la oferta de hortaliza y el precio de venta. En total se establecieron 9 escenarios de estudio estableciendo variaciones en la oferta de hortaliza $(\Delta 0)$ y variaciones en precio de venta $(\Delta \mathrm{P})$. En la

Tabla 1 se pueden ver los escenarios establecidos.

Tabla 1.Escenarios de estudio a partir de parámetros de perturbación

\begin{tabular}{|l|rr}
$\begin{array}{c}\text { ESCENARIO } \\
(\mathbf{E})\end{array}$ & $\begin{array}{c}\text { Variación en } \\
\text { el precio } \\
(\boldsymbol{\Delta P})\end{array}$ & $\begin{array}{c}\text { Variación en } \\
\text { la oferta } \\
(\mathbf{\Delta} \mathbf{0})\end{array}$ \\
\hline E1 & $0 \%$ & $100 \%$ \\
E2 & $13 \%$ & $93 \%$ \\
E3 & $25 \%$ & $85 \%$ \\
E4 & $38 \%$ & $78 \%$ \\
E5 & $50 \%$ & $70 \%$ \\
E6 & $63 \%$ & $63 \%$ \\
E7 & $75 \%$ & $55 \%$ \\
E8 & $88 \%$ & $48 \%$ \\
E9 & $100 \%$ & $40 \%$ \\
\hline
\end{tabular}

En total se estableció una variación del 0\% al 100\% del rango de los precios mínimos y máximos de ventas de las diferentes hortalizas $(\Delta \mathrm{P})$, de igual forma se decidió que los productores podía reducir su producción hasta un $40 \%$ de sus capacidad máximas en condiciones de alta cosecha $(\Delta O)$.

\section{Definición característica de operación $(\beta)$}

En total se establecieron cinco (5) características de operación para medir el funcionamiento de la cadena a nivel operativo estos son: La configuración de la cadena ( $\beta 1)$, el porcentaje de utilización de los puntos de Cross docking ( $\beta 2)$, el porcentaje de utilización de centros de distribución ( $\beta 3$ ), y el porcentaje de utilización de vehículos ( $\beta 4)$. La primera característica $(\beta 1)$ esta dada por el número de centros de acopio, centros de distribución y de vehículos que se requieran abrir y utilizar para el abastecimiento. La característica de operación ( $\beta 2$ ) correspondiente al porcentaje de utilización de brócoli y cilantro buscando la mayor retribución económica (Esto como se describe en la Fig. 1).

los puntos de Cross docking se calcula empleando la siguiente ecuación:

$$
\beta 2_{k}=\left[\sum_{i} \sum_{j} \sum_{v o} \sum_{t}\left[\frac{X O_{i, j, k, v o, t}}{C C R_{k} * A 1_{k}}\right] /{ }_{12}\right] * 100 \%
$$

La característica de operación ( $\beta 3$ ) correspondiente al porcentaje de utilización de los puntos de centros de distribución se calcula empleando la siguiente ecuación:

$$
\beta 3_{l}=\left[\sum_{i} \sum_{k} \sum_{v p} \sum_{t}\left[\frac{X P_{i, k, l, v p, t}}{C C D_{l} * A 2_{l}}\right] /{ }_{12}\right] * 100 \%
$$

En cuanto a la característica de operación ( $\beta 4)$ respecto al porcentaje de utilización de los vehículos se estableció un indicador para el transporte entre los diferentes eslabones de la cadena, como se muestra a continuación:

$$
\begin{aligned}
& \beta 41=\left[\frac{\sum_{i} \sum_{j} \sum_{k} \sum_{v o} \sum_{t} X O_{\mathrm{i}, \mathrm{j}, \mathrm{k}, \mathrm{vo}, \mathrm{t}}}{\sum_{v o} K T 1_{v o} * B O_{v o}} / 12\right] * 100 \% \\
& \beta 42=\left[\frac{\sum_{i} \sum_{k} \sum_{l} \sum_{v p} \sum_{t} X P_{\mathrm{i}, \mathrm{k}, \mathrm{l}, \mathrm{vp}, \mathrm{t}}}{\sum_{v p} K T 2_{v p} * B P_{v p}} /{ }_{12}\right] * 100 \% \\
& \beta 43=\left[\frac{\sum_{i} \sum_{l} \sum_{m} \sum_{v q} \sum_{t} X Q_{\mathrm{i}, \mathrm{l}, \mathrm{m}, \mathrm{vq}, \mathrm{t}}}{\sum_{v q} K T 3_{v q} * B Q_{v q}} / 12\right] * 100 \% \\
& \beta 45=\left[\frac{\sum_{i} \sum_{l} \sum_{n} \sum_{v r} \sum_{t} X R_{\mathrm{i}, \mathrm{ln}, \mathrm{vr}, \mathrm{t}}}{\sum_{v r} K T 4_{v r} * B R_{v r}} / 12\right] * 100 \% \\
& \beta 46=\left[\frac{\sum_{i} \sum_{k} \sum_{v t} \sum_{t} X T_{\mathrm{i}, \mathrm{k}, \mathrm{vt}, \mathrm{t}}}{\sum_{v t} K T 5_{v t} * B T_{v t}} / 12\right] * 100 \%
\end{aligned}
$$

Se tiene en total cinco (5) indicadores, uno para los vehículos empleados para el transporte entre los productores y los puntos de cros docking ( $\beta 41)$, otro para los vehículos empleados para el transporte entre los puntos de Cross docking y los centros de distribución ( $\beta 42)$, otro para los vehículos del transporte entre los centros de distribución hasta los fruvers ( $\beta 43)$, uno para el transporte desde los centros de distribución hasta los almacenes de 
grandes superficies $(\beta 44)$ y uno para el transporte entre los puntos de Cross docking hasta Corabastos $(\beta 45)$. Estos se calculan con las ecuaciones mostradas a continuación:

\section{Definición requerimiento de robustez $(\pi)$}

Se establecieron dos requerimientos de robustez el primero referente a la utilidad que tiene el sistema y el segundo las emisiones de Co2 de los vehículos que se utilizan para el transporte de los productos entre los diferentes eslabones. El primero parámetro correspondiente a la utilidad $(\pi 1)$, se calcula empleando la ecuación (1) que se utilizó para el cálculo de la función objetivo:

$$
\pi 1=F O=I N C-C T O
$$

En cuanto al requerimiento de robustez $(\pi 2)$ se emplea la ecuación (32), la cual, calcula la totalidad de gramos de $\mathrm{CO} 2$ que se pueden emitir si se compran y utilizan vehículos para el transporte entre los diferentes eslabones.

$$
\begin{aligned}
\pi 2=E C O=\sum_{i}^{I} & \sum_{j}^{J} \sum_{k}^{K} \sum_{v o}^{V O} \sum_{t}^{T}\left(C O 1_{v o} * X O_{i, j, k, v o, t} * D J K_{j k}+\sum_{i}^{I} \sum_{k}^{K} \sum_{l}^{L} \sum_{v p}^{V P} \sum_{t}^{T} C O 2_{v p} * X P_{i, k, l, v p, t} * D K L_{k l}\right. \\
& +\sum_{i} \sum_{l}^{L} \sum_{m}^{M} \sum_{v q}^{V Q} \sum_{t}^{T}\left(C O 3_{v q} * X Q_{i, l, m, v q, t}\right)+D L M_{l m}+\sum_{i}^{I} \sum_{l}^{L} \sum_{n}^{N} \sum_{v r}^{V R} \sum_{t}^{T}\left(C O 4_{v r} * X R_{i, l, n, v r, t}\right) * D L N_{l n} \\
& +\sum_{i}^{I} \sum_{k}^{K} \sum_{v t}^{V T} \sum_{t}^{T}\left(C O 5_{v t} * X T_{i, k, v t, t}\right)+D K C_{k}
\end{aligned}
$$

Es importante resaltar que las emisiones de $\mathrm{CO} 2$ se tienen en cuenta como segundo requerimiento de robustez que tener en cuenta para de decisión de la configuración de la cadena, dado que el principal objetivo es la maximización de utilidades para los 19 productores involucrados en el estudio.

\section{Identificación de configuraciones}

Para identificar las configuraciones de la cadena de suministro hortícola se realizaron las corridas en

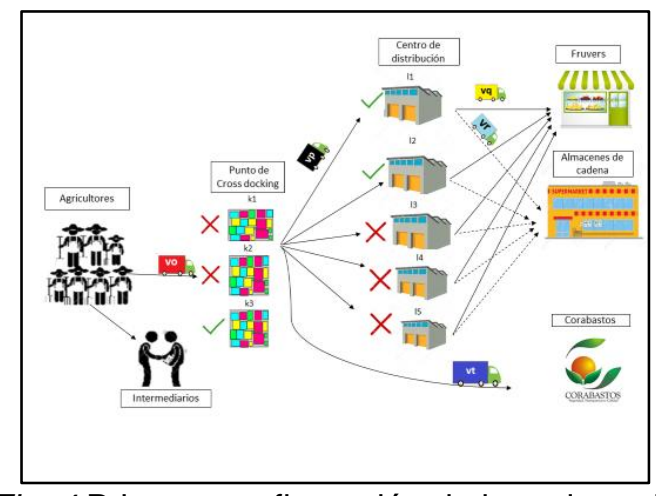

Fig. 4.Primera configuración de la cadena de suministro
Gams de los nueve (9) escenarios que se plantearon en la fase uno del análisis de robustez. Tras la corrida de los nueve (9) escenarios se identificaron siete (7) configuraciones de la cadena hortícola, esto teniendo en cuenta que durante algunos escenarios se pueden abrir los mismos puntos de Cross Docking, centros de distribución y emplear la misma cantidad de vehículos con igual capacidad. Las configuraciones de la cadena de suministro se representan en las figuras 4 a 10 .

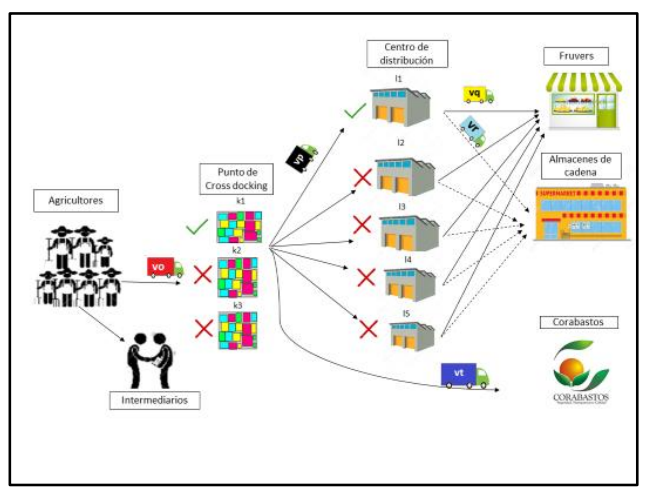

Fig. 5.Segunda configuración de la cadena de suministro 


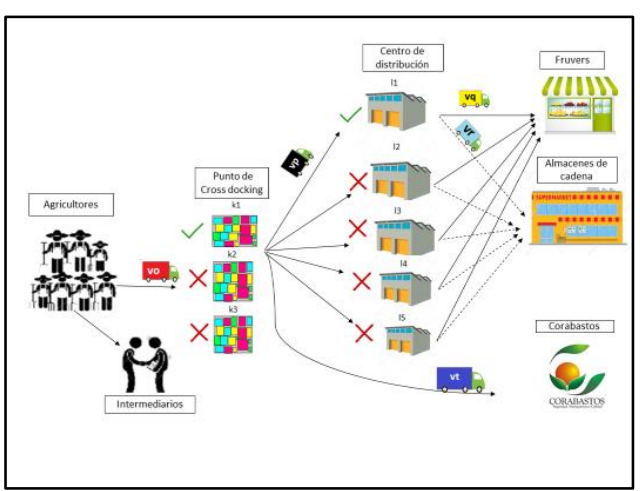

Fig. 6. Tercera configuración de la cadena de suministro

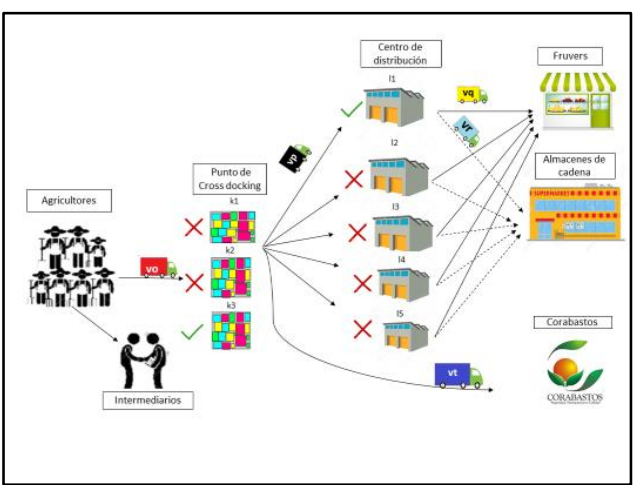

Fig. 8.Quinta configuración de la cadena de suministro

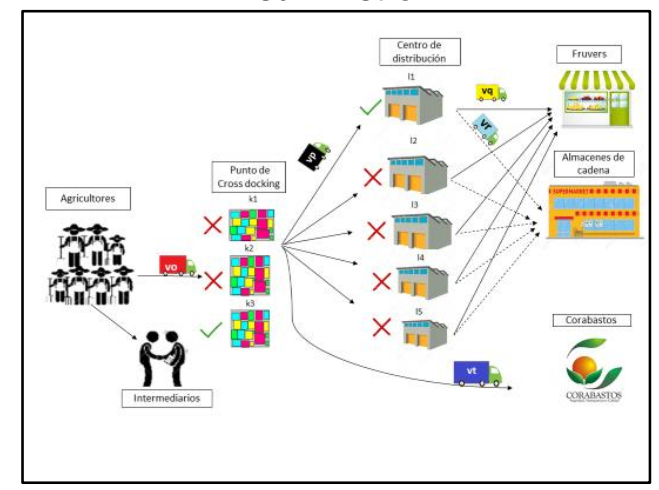

Fig. 10.Septima configuración de la cadena de suministro

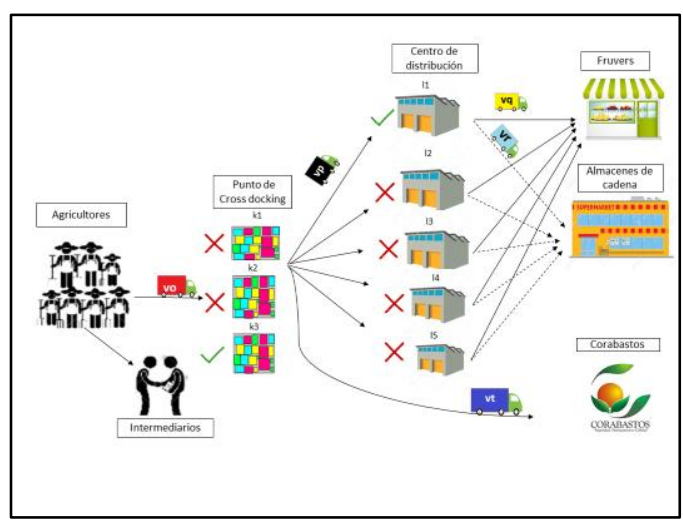

Fig. 7.Cuarta configuración de la cadena de suministro

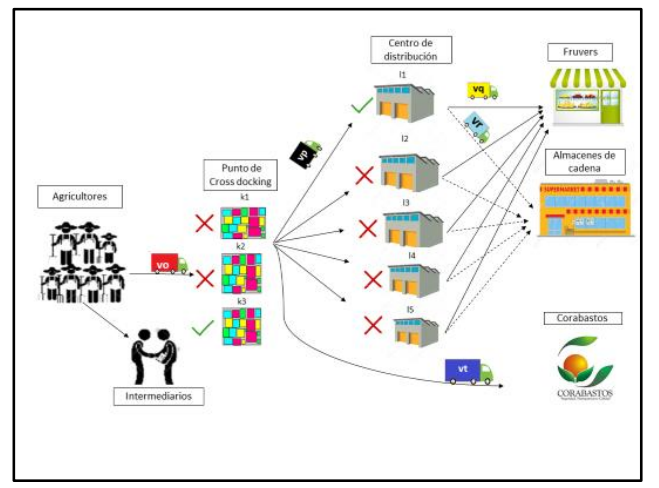

Fig. 9.Sexta configuración de la cadena de suministro

La descripción de los tipos de transporte que se deben adquirir y su cantidad, se ven representados a continuación: 
Tabla 2.Vehículos requeridos para las diferentes configuraciones de cadena

\begin{tabular}{|c|c|c|c|c|c|c|}
\hline Configuración & $\begin{array}{l}\text { descripción } \\
\text { transporte }\end{array}$ & $\begin{array}{c}\text { Agricultores } \\
\text { a puntos de } \\
\text { Cross } \\
\text { docking }\end{array}$ & $\begin{array}{c}\text { Cross } \\
\text { docking a } \\
\text { centros de } \\
\text { distribución }\end{array}$ & $\begin{array}{l}\text { Centros de } \\
\text { distribución } \\
\text { a Fruvers }\end{array}$ & $\begin{array}{c}\text { Centros de } \\
\text { distribución } \\
\text { a } \\
\text { almacenes } \\
\text { de cadena }\end{array}$ & $\begin{array}{c}\text { Cross } \\
\text { docking a } \\
\text { Corabastos }\end{array}$ \\
\hline \multirow{2}{*}{$\begin{array}{c}\text { Primera } \\
\text { Configuración }\end{array}$} & $\begin{array}{l}\text { Tipo de } \\
\text { vehículo }\end{array}$ & vo3 & vp3 & vq3 & vr3 & $\mathrm{N} / \mathrm{A}$ \\
\hline & Cantidad & 3 & 3 & 1 & 2 & $\mathrm{~N} / \mathrm{A}$ \\
\hline \multirow{2}{*}{$\begin{array}{l}\text { Segunda } \\
\text { Configuración }\end{array}$} & $\begin{array}{l}\text { Tipo de } \\
\text { vehículo }\end{array}$ & vo3 & vp3 & vq3 & $\begin{array}{l}\text { vr1 } \\
\text { vr3 }\end{array}$ & $\mathrm{N} / \mathrm{A}$ \\
\hline & Cantidad & 2 & 2 & 1 & $\begin{array}{l}1 \\
1\end{array}$ & $\mathrm{~N} / \mathrm{A}$ \\
\hline \multirow{2}{*}{$\begin{array}{c}\text { Tercera } \\
\text { Configuración }\end{array}$} & $\begin{array}{l}\text { Tipo de } \\
\text { vehículo }\end{array}$ & vo3 & vp3 & vq3 & vr1 & $\mathrm{N} / \mathrm{A}$ \\
\hline & Cantidad & 1 & 1 & 1 & 1 & $\mathrm{~N} / \mathrm{A}$ \\
\hline \multirow{2}{*}{$\begin{array}{c}\text { Cuarta } \\
\text { Configuración }\end{array}$} & $\begin{array}{l}\text { Tipo de } \\
\text { vehículo }\end{array}$ & vo3 & vp3 & vq3 & vr1 & vt3 \\
\hline & Cantidad & 2 & 1 & 1 & 1 & 1 \\
\hline \multirow{2}{*}{$\begin{array}{c}\text { Quinta } \\
\text { Configuración }\end{array}$} & $\begin{array}{l}\text { Tipo de } \\
\text { vehículo }\end{array}$ & $\begin{array}{l}\text { vo2 } \\
\text { vo3 }\end{array}$ & vp3 & vq3 & vr1 & vt3 \\
\hline & Cantidad & $\begin{array}{l}1 \\
1\end{array}$ & 1 & 1 & 1 & 1 \\
\hline \multirow{2}{*}{$\begin{array}{c}\text { Sexta } \\
\text { Configuración }\end{array}$} & $\begin{array}{l}\text { Tipo de } \\
\text { vehículo }\end{array}$ & $\begin{array}{l}\text { vo1 } \\
\text { vo3 }\end{array}$ & vp3 & vq2 & $\mathrm{N} / \mathrm{A}$ & vt3 \\
\hline & Cantidad & $\begin{array}{l}1 \\
1\end{array}$ & 1 & 1 & $\mathrm{~N} / \mathrm{A}$ & 1 \\
\hline \multirow{2}{*}{$\begin{array}{c}\text { Séptima } \\
\text { Configuración }\end{array}$} & $\begin{array}{l}\text { Tipo de } \\
\text { vehículo }\end{array}$ & vo3 & vp3 & vq2 & $\mathrm{N} / \mathrm{A}$ & vt3 \\
\hline & Cantidad & 1 & 1 & 1 & $\mathrm{~N} / \mathrm{A}$ & 1 \\
\hline
\end{tabular}

Con el propósito de mostrar la cantidad de producto que se abastece en los diferentes eslabones de la cadena, a partir de los escenarios planteados inicialmente, se construyó la Fig. 11. Esta muestra el porcentaje de demanda que se puede satisfacer en los diferentes escenarios eslabones de la cadena que demanda hortalizas. Es importante tener en cuenta que estás se construyeron con la misma información que permitió detectar las diferentes configuraciones, por lo tanto, no se tiene en cuenta las configuraciones en este análisis. 


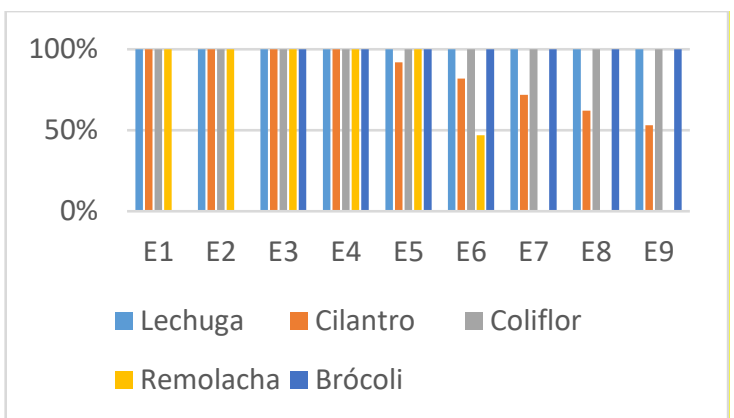

\section{A. Fruvers}

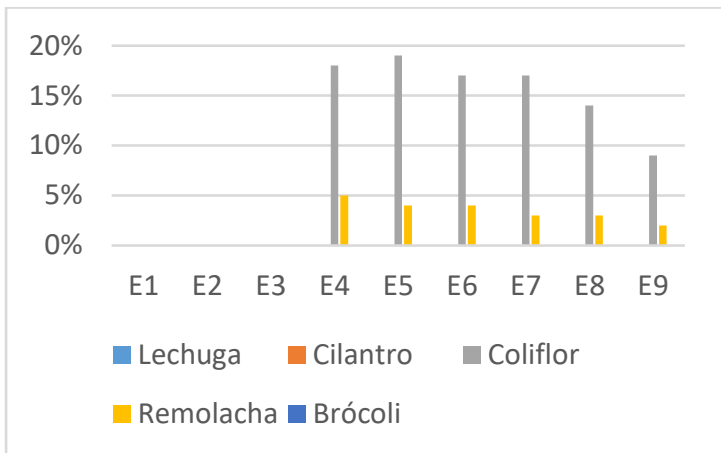

C. Corabastos

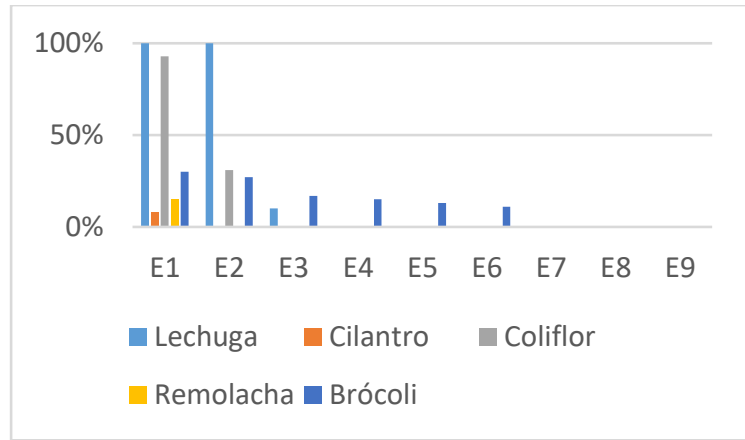

B. Almacenes de cadena

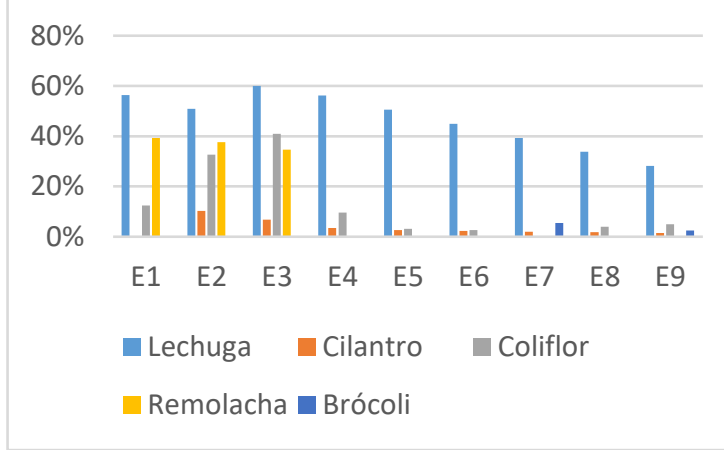

D. Intermediarios

Fig. 11.Demanda satisfecha en los diferentes puntos de demanda

Como se observa en la Fig. 11 se puede ver que el eslabón donde más se abastece hortalizas son los fruvers (a) en donde la mayor parte de las hortalizas pueden ser entregadas en un $100 \%$ de acuerdo con la demanda. Por otro lado, es eslabón al que menos se le debe abastecer hortalizas es Corabastos (c) a este solo conviene entregarle coliflor y una porción de la producción de remolacha. A los eslabones almacenes de cadena e intermediarios no es conveniente entregarles la totalidad de los productos; la Fig. 11 (b) sugiere que solo se debe entregar a los almacenes de cadena un porcentaje del producto de lechuga durante el escenario e1 y e2 que es cuando existen altos niveles de producción y un precio de venta bajo; la lechuga es conveniente entregarla a los intermediarios (d) durante los diferentes escenarios de estudio, sin embargo esto solo puede ocurrir si se bastece primero la demanda de la lechuga de los fruvers (a).

\section{Evaluación del impacto de los parámetros de perturbación $(\propto)$ en las características de operación $(\beta)$}

Una vez identificadas las configuraciones de la cadena se procede a realizar el análisis del impacto de los perturbación $(\alpha)$ en las características de operación $(\beta)$,esto se hace a partir de la interpretación de las figuras. 


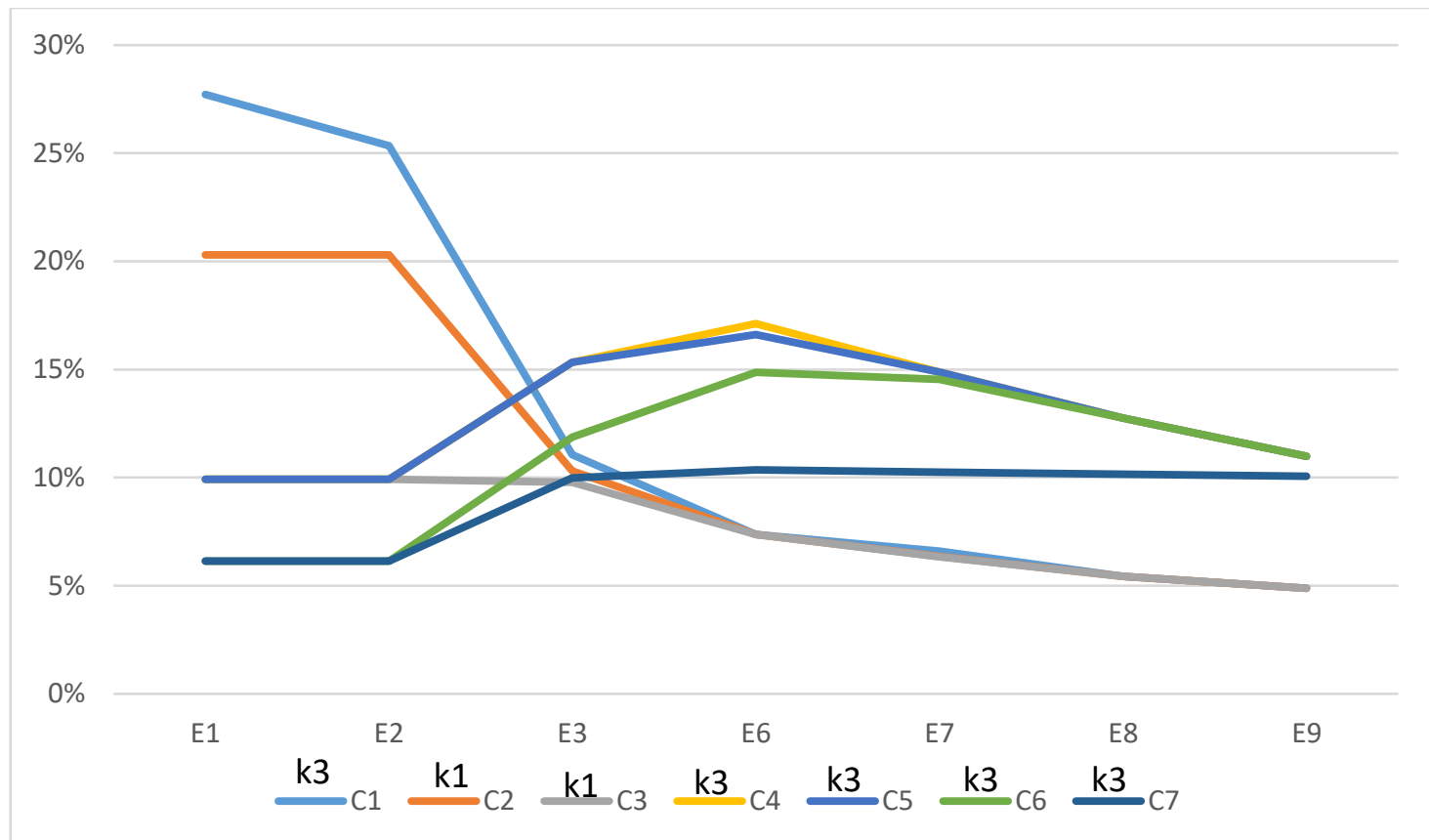

Fig. 12. Comportamiento del porcentaje de utilización de los puntos de Cross docking $\beta 2$

En la Fig. 12 se puede ver el comportamiento de la característica $\beta 2$. Desde el punto de vista operacional, la configuración 1 es considera la más robusta durante los escenarios de estudio e1 y e2 pero deja de ser robusta en los demás escenarios, debido a que deja de utilizar el punto de Cross docking k3, lo cual, no es conveniente para los productores debido a que abrirlo requiere una importante inversión. Las configuraciones c4 y c5 son consideradas las más robustas en términos operacionales; si bien no se utilizan demasiado durante el escenario e1 y e2 son las que mayor porcentaje de utilización presenta en los escenarios de estudio a un sabiendo que durante estos escenarios la producción es menor.

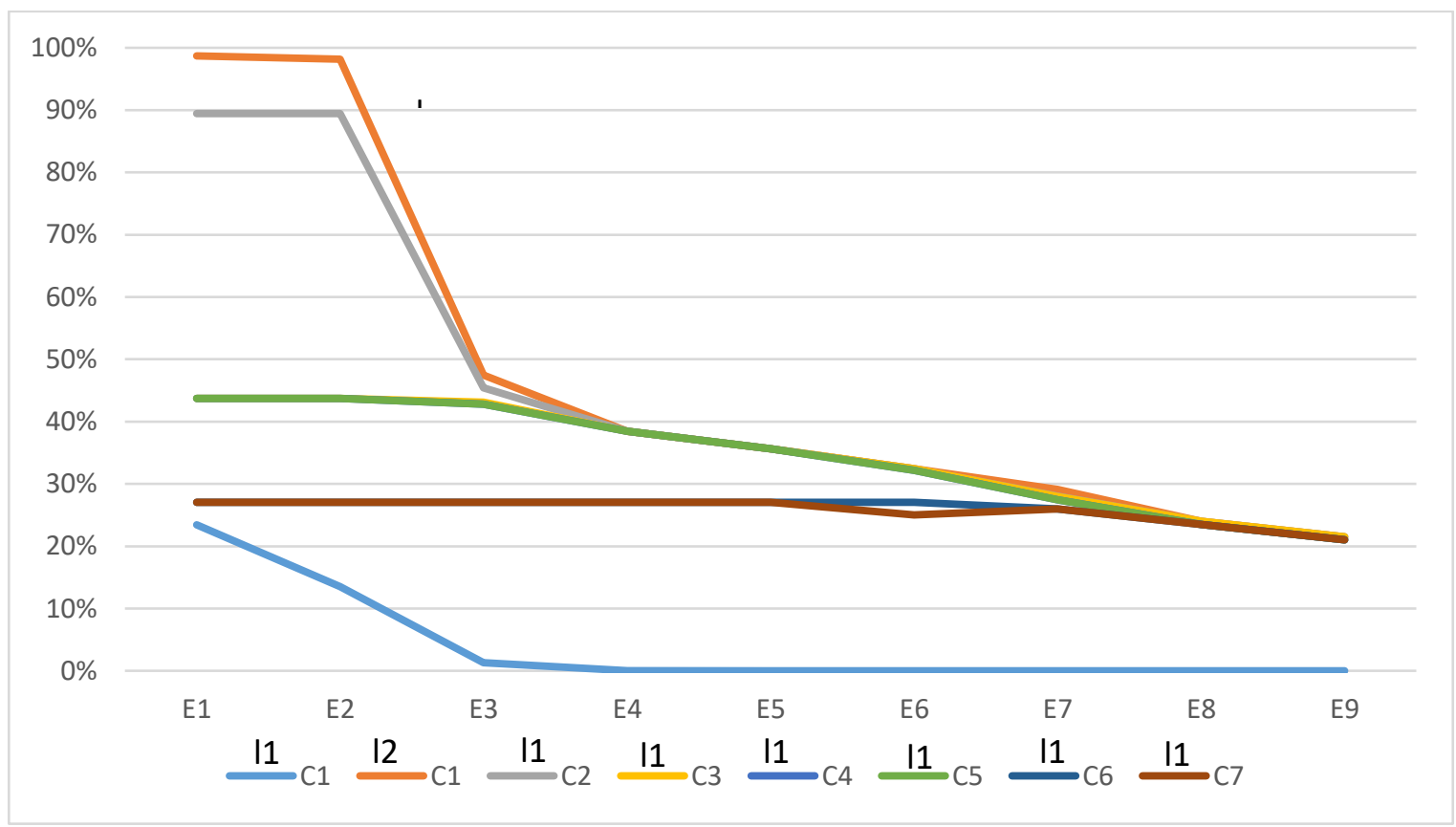

Fig. 13. Comportamiento del porcentaje de utilización de los centros de distribución $\beta 3$ 
En la figura se puede ver el comportamiento de la característica de operación $\beta 3$; para la configuración 1 se debe usar dos centros de distribución l1 y l2, se puede notar que la configuración 1 usando el centro de distribución I2 es la configuración más robusta, sin embargo, el centro de distribución I1 tiene un bajo porcentaje de utilización en los primeros escenarios y no se utiliza desde el escenario tres, lo cual no resulta conveniente para los productores pues en la mayoría de los escenarios solo usarían un centro de distribución . Dado esto, la configuración más robusta es c2, pues en comparación con las demás configuraciones, es la que mayor porcentaje de utilización presenta.

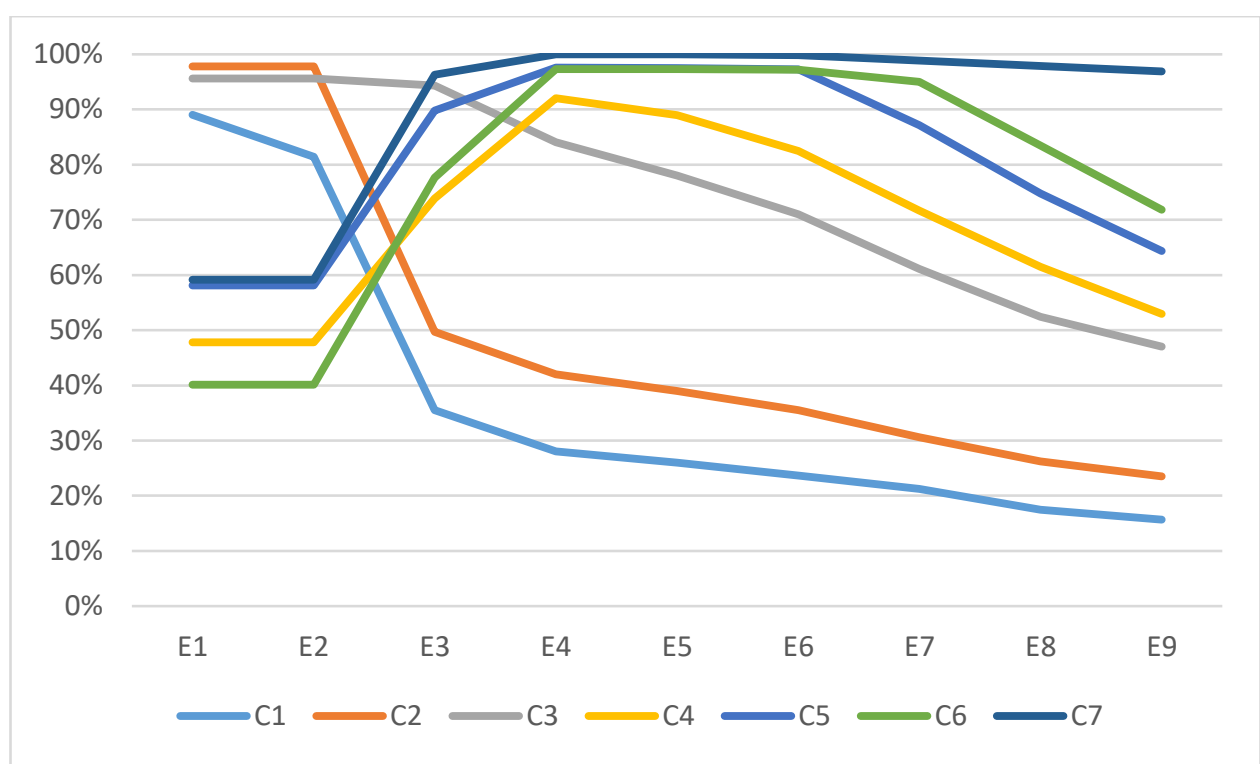

Fig. 14. Comportamiento del porcentaje de utilización de los vehículos vo $\beta 41$

El comportamiento de la característica de operación $\beta 41$, se puede evidenciar en la Fig. 14. La configuración c7 es considerada la configuración más robusta desde el punto de vista operacional, pues los vehículos tipo "vo" en los diferentes escenarios presentan un porcentaje de utilización alto, siendo así que desde el escenario tres se presenta un porcentaje muy cercano al $100 \%$ a un sabiendo que a partir de este escenario hasta escenario e9 la producción de hortalizas disminuye. 


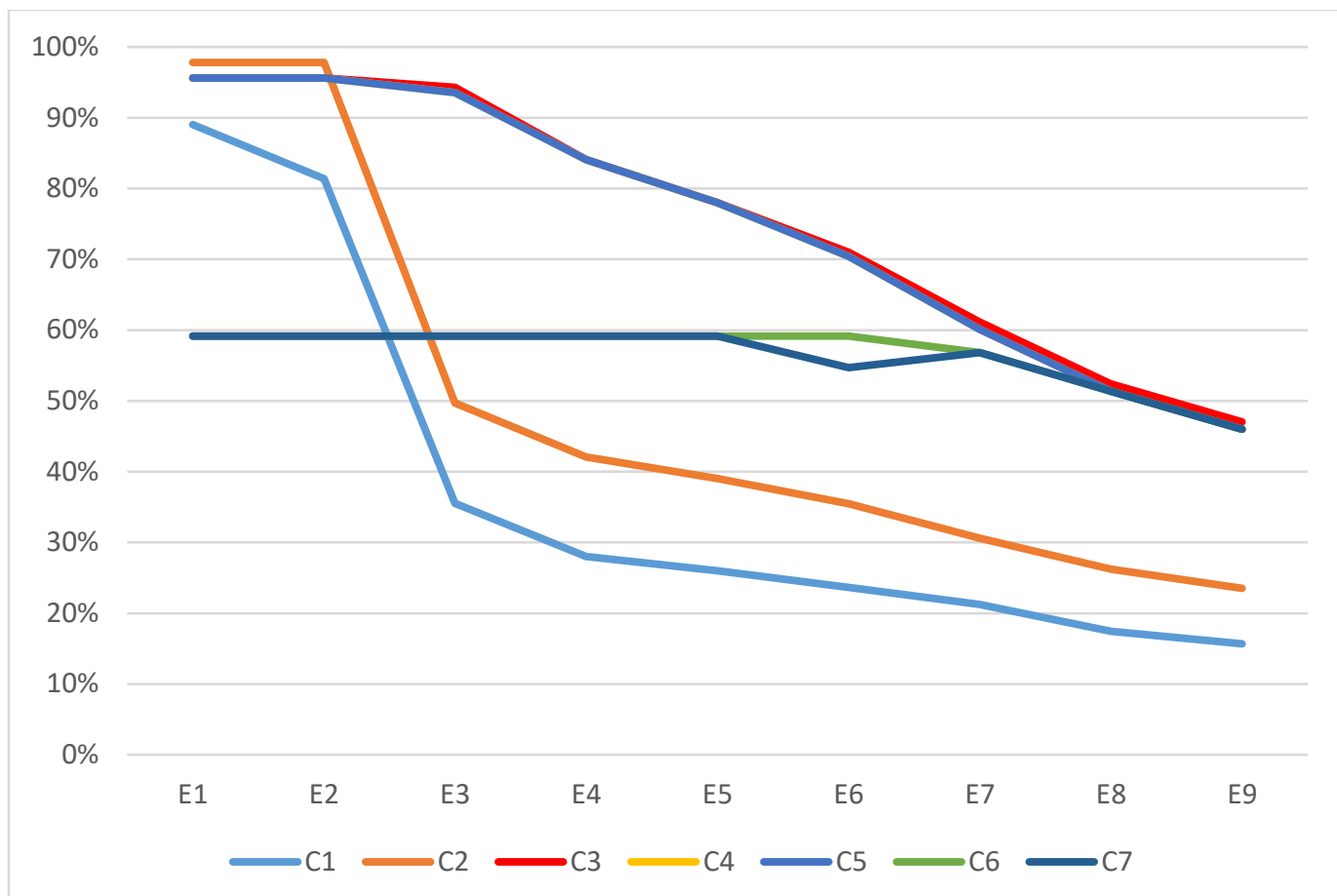

Fig. 15. Comportamiento del porcentaje de utilización de los vehículos vp $\beta 42$

El comportamiento de la característica operación $\beta 42$ se ilustra en la Fig. . Como se observa en la figura, la configuración más robusta es la configuración c3 pues representa la mayor utilización de los vehículos tipo vp a través de los diferentes escenarios.

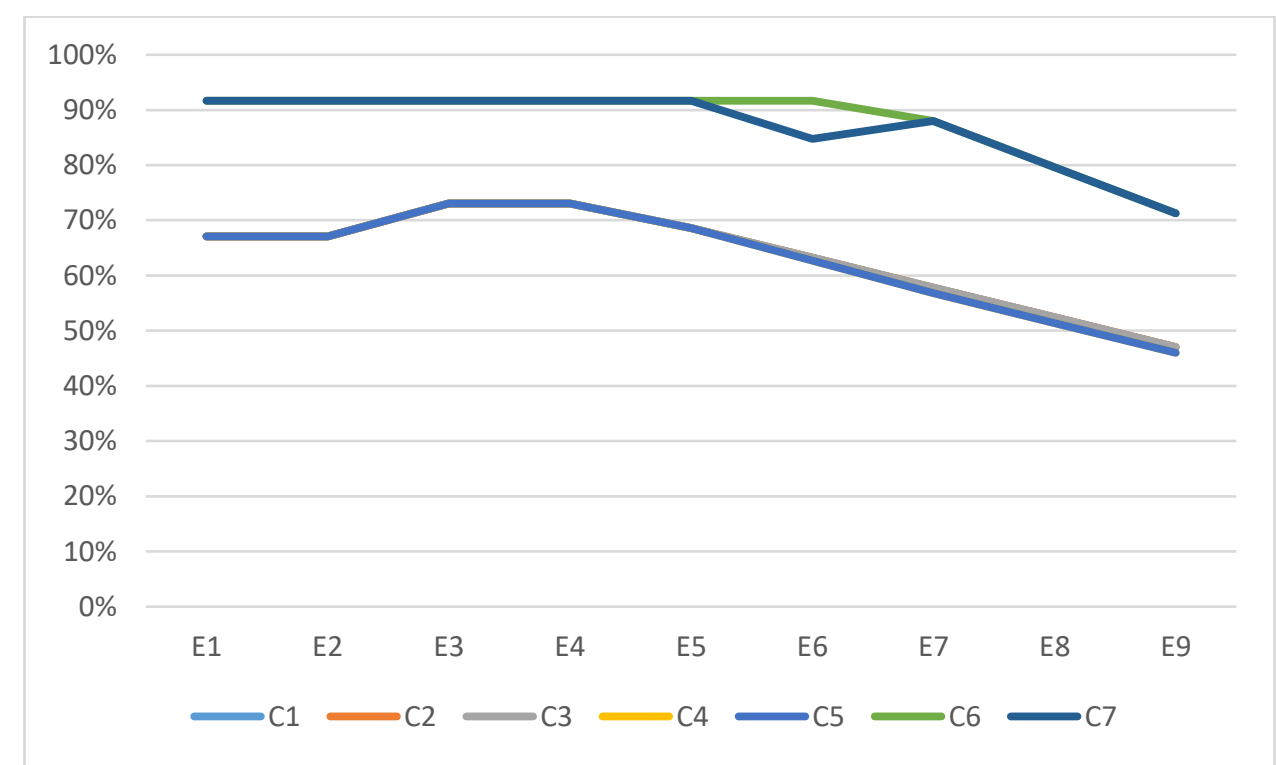

Fig. 16. Comportamiento del porcentaje de utilización de los vehículos vq $\beta 43$

El comportamiento de la característica de operación $\beta 43$ se muestra en la Fig. 16. Como se visualiza en la Figura, la configuración más robusta es c6 ya que representa la mayor utilización de los vehículos tipo vq en los diferentes escenarios. 


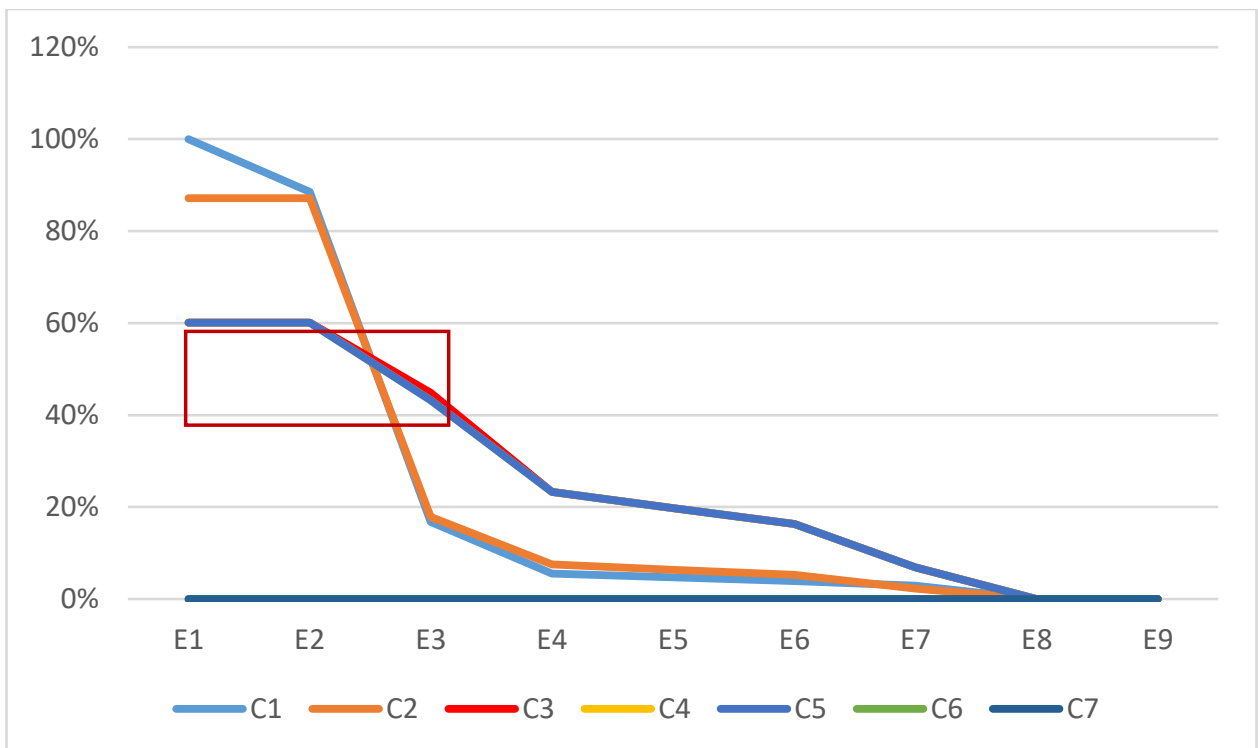

Fig.17. Comportamiento del porcentaje de utilización de los vehículos vr $\beta 44$

El comportamiento de la característica de operación $\beta 44$ se ilustra en la Fig. 17. Si bien la configuración c4 y c5 presentan diferente diferencias en los porcentajes de utilización en términos decimales, la configuración consideraba más robusta es la c3, la cual presenta el mayor porcentaje de utilización e3 como se resalta en la Figura.

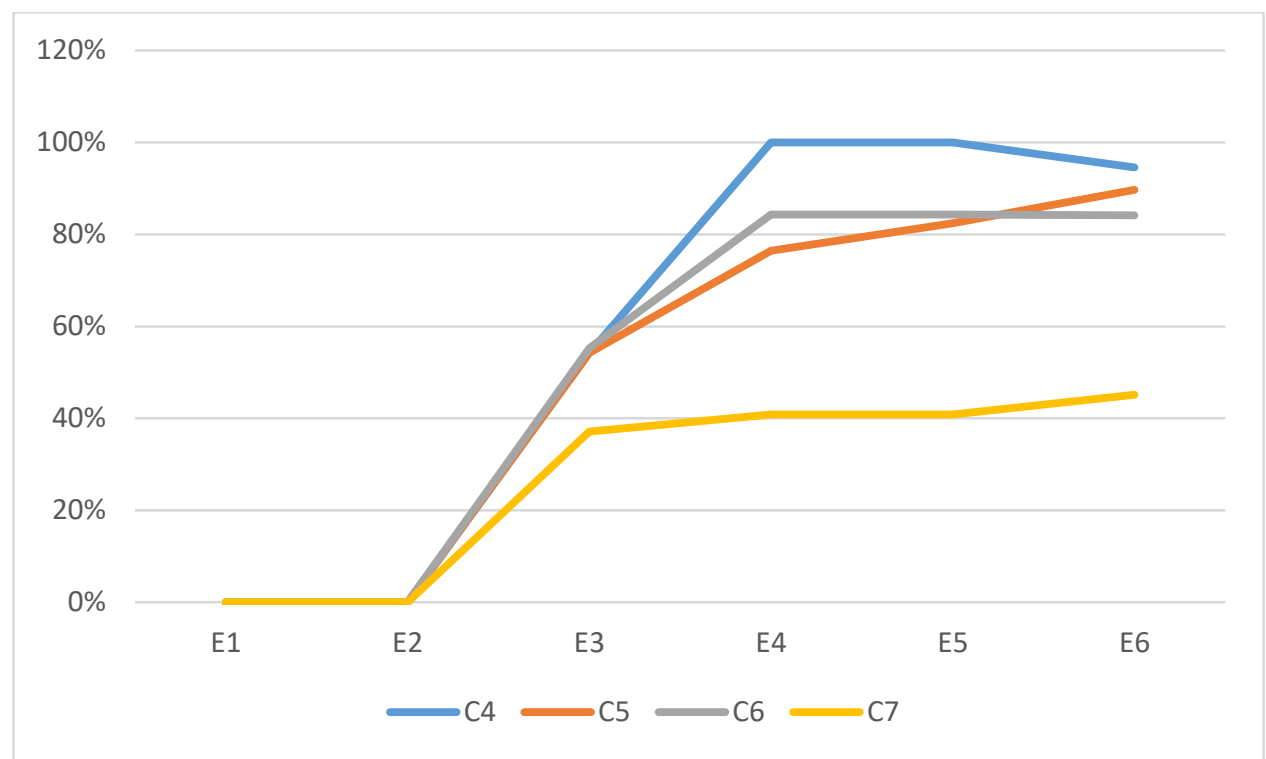

Fig. 18. Comportamiento del porcentaje de utilización de los vehículos vt $\beta 45$

En la Fig. 18 se muestra el comportamiento de la característica de operación $\beta 45$ es importante tener en cuenta que, solamente se incluyeron en esta figura las configuraciones $\mathrm{c} 4, \mathrm{c} 5, \mathrm{c} 6$ y $\mathrm{c} 7$. Esto se debe a que las configuraciones c1, c2 y c3 indicaron como resultado que no se deben adquirir vehículos para el abastecimiento hasta Corabastos.
Interpretando los resultados obtenidos en la figura es posible decir que la configuración que presenta mayor robustez es la c4 debido a que presenta en mayor porcentaje de utilización durante los diferentes escenarios de estudio principalmente en los escenarios e4, e5 y e6. 
Evaluación del impacto de los parámetros de perturbación $(\propto)$ en los requerimientos robustez $(\pi)$

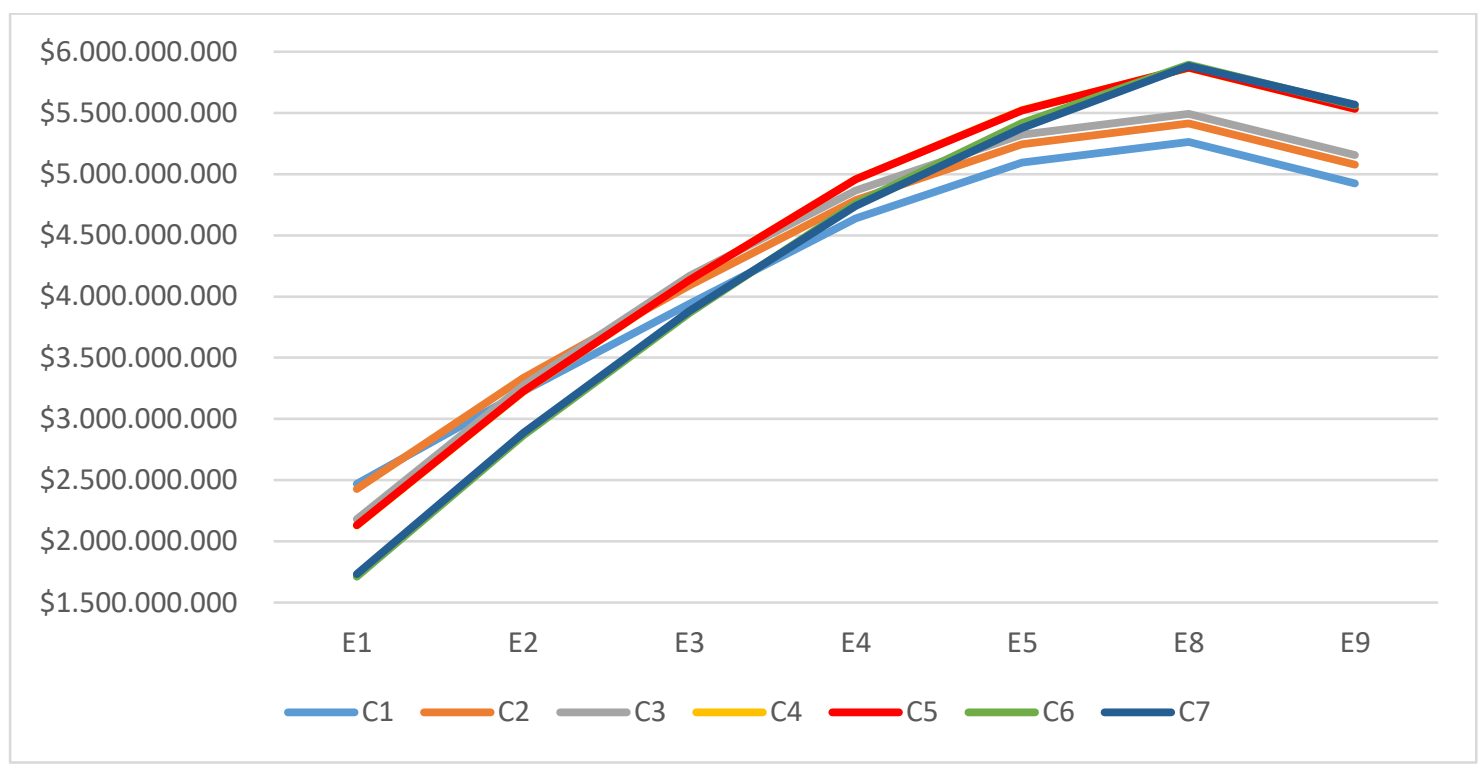

Fig. 19. Comportamiento del requerimiento de robustez $\pi 1$

En el comportamiento del requerimiento de robustez $\pi 1$ se puede observar en la Fig. 19. Si bien la configuración c4 y c5 permiten las mayores utilidades durante los diferentes escenarios de estudio, es considerada la configuración C5 la más robusta, debido a que en promedio permite obtener $\$ 1$,
288,571 de utilidad más que la configuración c4 (este valor se comparó a partir del promedio de las utilidades de las diferentes configuraciones).Con el propósito de evidenciar la cadena más robusta desde el parámetro se construyó la Fig. 20.

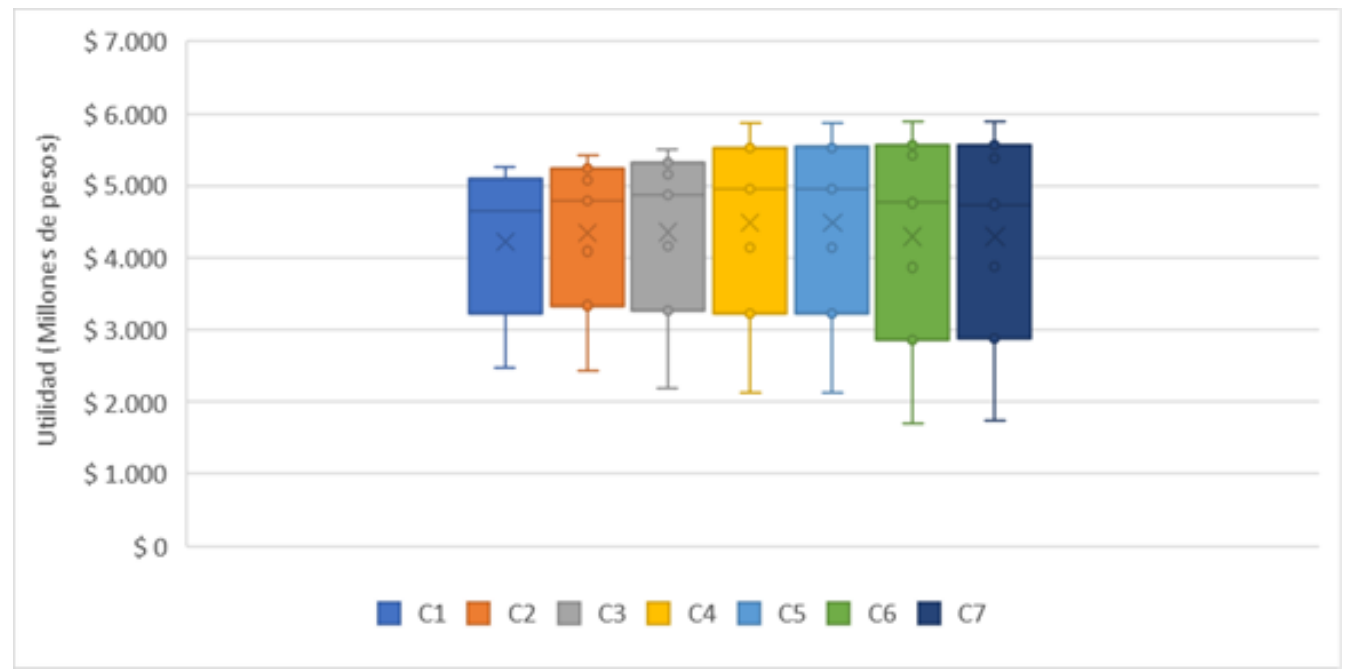

Fig. 20. Variación de la utilidad por configuración

Como se puede ver en la figura la configuración que presenta menos variación es la configuración 1; sin embargo, al concepto que se defino de robustez se entiende que la configuración más robusta del presente estudio es C5 debido a que es la que presenta un mayor rendimiento durante su operación en los diferentes escenarios evaluados. Su media de la utilidad se encuentra por encima de la media de las utilidades de las demás configuraciones. 


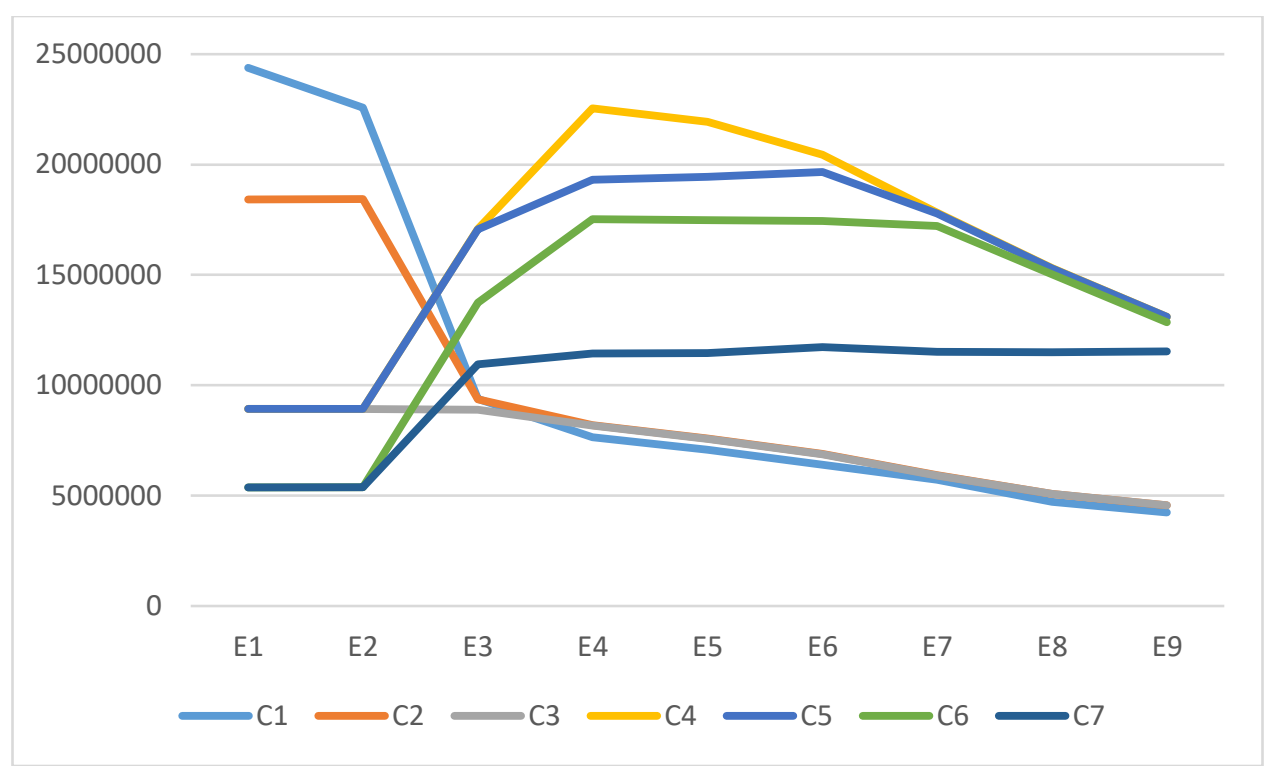

Fig. 21. Comportamiento del requerimiento de robustez $\pi 2$

El comportamiento del requerimiento de robustez $\pi 2$ se puede observar en la Fig. 21.Se puede notar que la configuración más robusta es la configuración C3, pues representa la menor cantidad de emisiones de Co2 de los diferentes escenarios de estudio, esto teniendo en cuenta que en este caso el rendimiento del sistema se mide a partir de las emisiones para lo cual la configuración que menos emisiones genere es la que se considera más robusta. Esto se puede ver de forma más clara en la Fig. 22 donde se percibe que la configuración c3 es la que menor cantidad de Co2 emite durante todos los escenarios y a su vez es la menor variaciones de Co2 presenta.

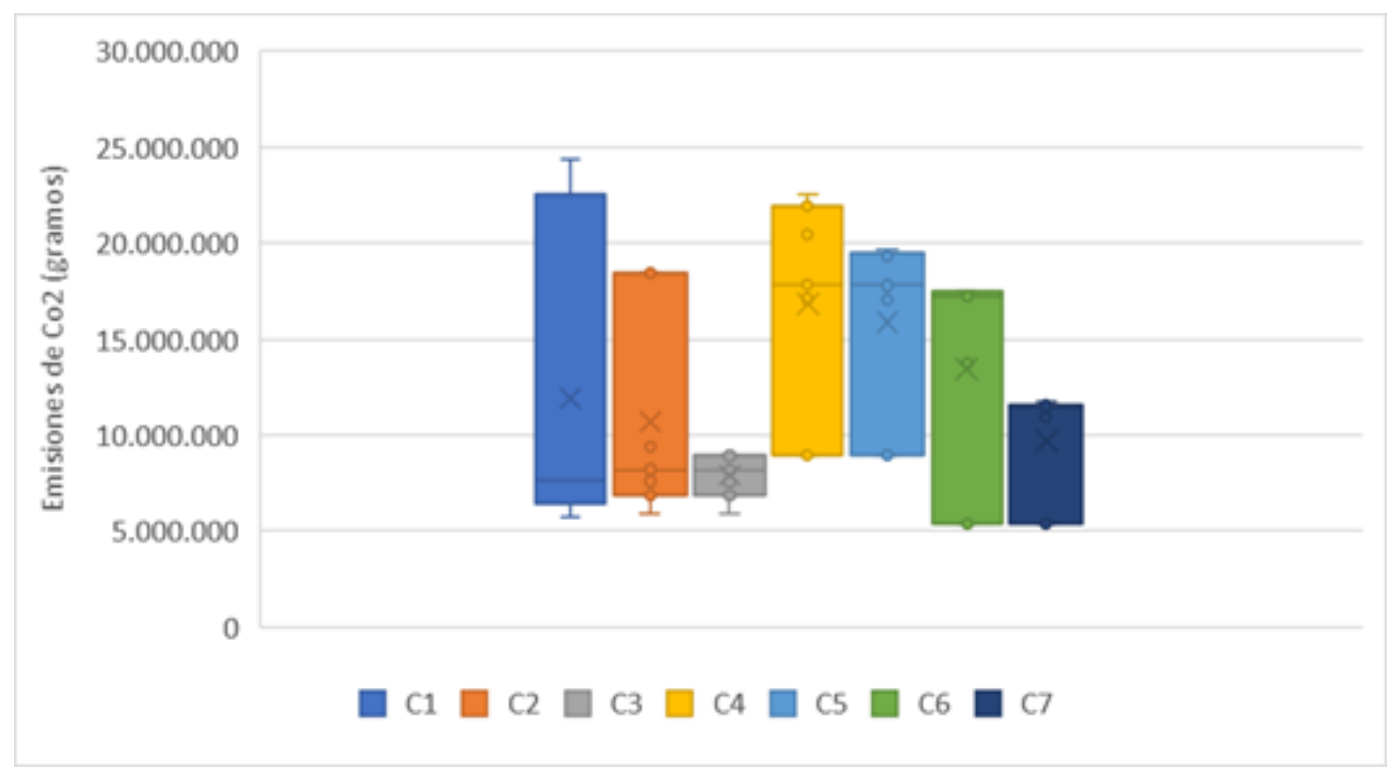

Fig. 22. Variación de las emisiones de Co2 por configuración

En este punto se tienen resultados contradictorios por un lado la configuración c5 es la mayor utilidad sin embargo no es la menor generadora de emisiones de Co2.Por otro lado la configuración c3 es la adecuada en términos de emisiones de $\mathrm{CO} 2$ pero no es la más conveniente en cuanto a la generación de utilidades. Para poder determinar cuál de las dos configuraciones es la más conveniente se debe tener 
en cuenta los requerimientos de los productores. En este caso acorde con el objetivo planteado con el presente estudio, se pretende el diseño de la cadena de suministro hortícola que genere el mayor beneficio, por lo tanto, se establece como configuración para los productores la configuración
C5. Esta configuración se muestra en la figura siguiente:

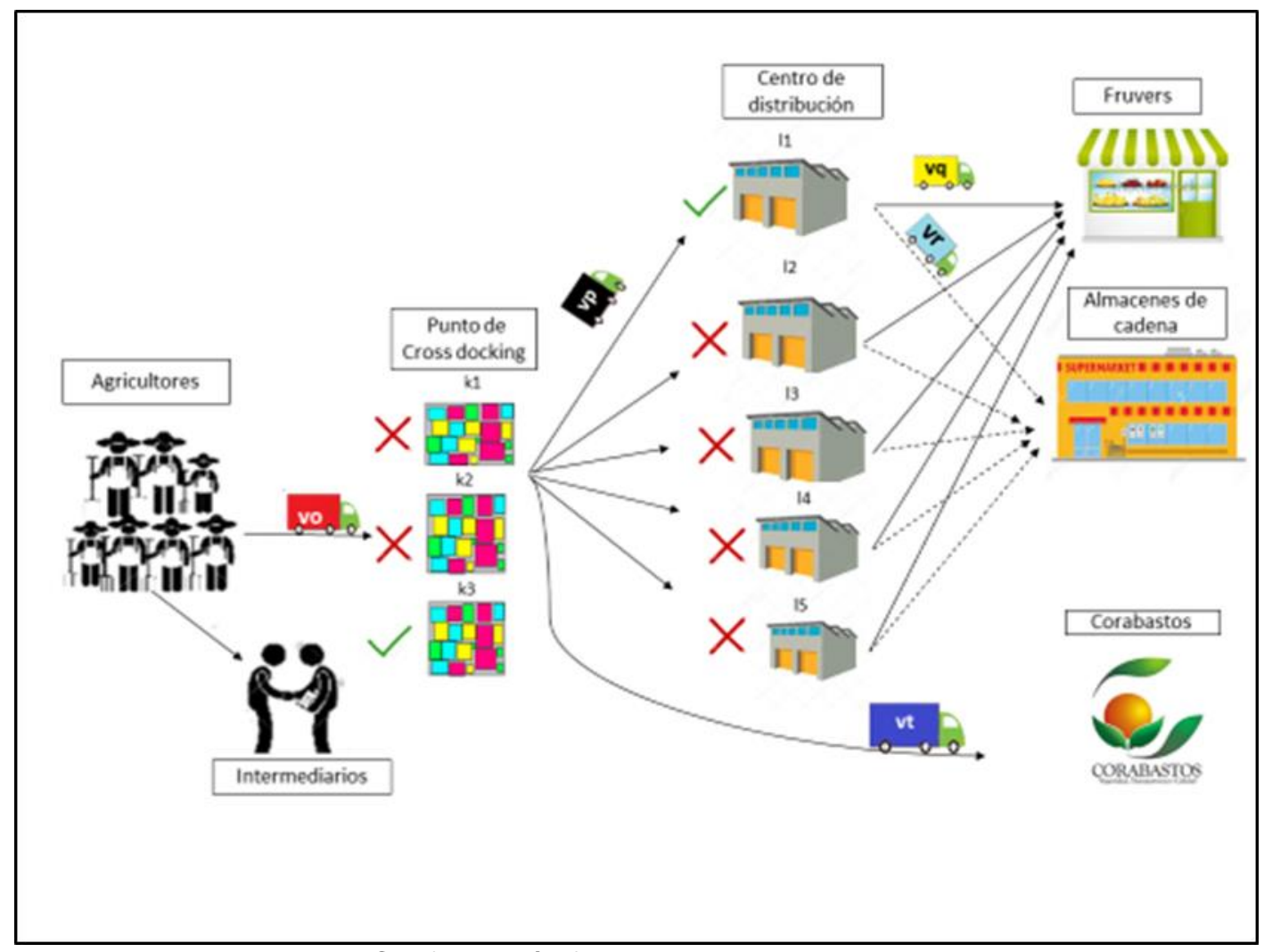

Fig.23. Configuración final de la cadena de suministro

La decisión que se debe tomar es abrir el punto de Cross docking $k 3$, el centro de distribución 11.Adicionalmete se deben comprar seis (6) vehículos los cuales servirán para abastecer hortalizas a los diferentes clientes, estos son :Dos (2) vehículos uno con capacidad de 4.7 ton y el otro con capacidad de 3 ton ,un vehículo de 4,7 ton para el transporte de las hortalizas desde los puntos de Cross docking hasta centros de distribución, un (1) vehículo con capacidad de 4.7 ton para el abastecimiento de productos desde los centros de distribución hasta los fruvers, un (1) vehículo de capacidad de 2.2 ton para transportar hortalizas desde el centro de distribución hasta los almacenes de cadena y un vehículo con capacidad de 4.7 ton para el transporte de productos desde los puntos de Cross docking hasta Corabastos.

Adicional a lo dicho anteriormente se muestra con fines ilustrativos en la Fig. 24 el porcentaje de demanda satisfecha en los diferentes eslabones 


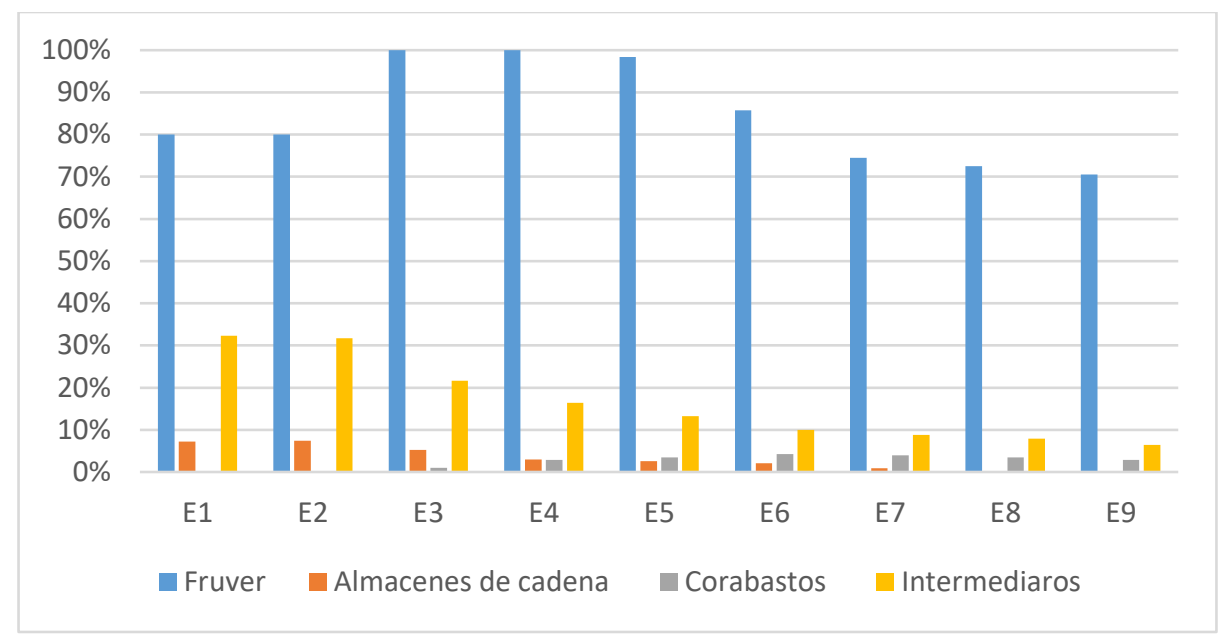

Fig.24. Demanda satisfecha con la Configuración C5

Como se puede ver en la mayoría de los escenarios se satisface más del $70 \%$ de la demanda a los fruvers lo cual sugiere que es muy conveniente abastecer directamente a este eslabón. Por otro lado, se tiene que durante todos los escenarios se debe vender hasta una porción importante de hortalizas a los intermediarios lo que indica que debe existir un contacto con los intermediarios para vender ocasionalmente el producto. En cuanto a la demanda satisfecha en Corabastos, no se cumple con más del $10 \%$ en todos los escenarios principalmente en los escenarios en los que existe una oferta baja con precios altos de las hortalizas, además, no es conveniente venderle a Corabastos en los escenarios (e7, e8 y e9).Por otra parte los porcentaje de demanda satisfecha en los almacenes de cadena no supera el $10 \%$, lo que indicaría que no se le debería abastecer a este eslabón, esto bajo el argumento de que los almacenes de cadena solicitan que se les mantenga unos mínimos de oferta durante un tiempo determinado y esto no es conveniente para los productores debido a que podrían generar mayor utilidad en otros eslabones.

\section{CONCLUSIONES}

Según la caracterización que se realizó se determinó que en el municipio de Cajicá se producen 14 diferentes tipos de hortalizas dentro de los que se encuentra la lechuga, brócoli coliflor, cilantro, espinaca, zanahoria, remolacha, arveja, papa, perejil, apio, acelga, calabacín y ajo, de estos catorce productos se escogieron cinco, la lechuga, el cilantro, coliflor, brócoli y remolacha, que de acuerdo al análisis de Pareto y a lo manifestado por los agricultores son los productos más representativos. Se estableció que el $95 \%$ de los agricultores involucrados en el estudio cultivan lechuga siendo este el producto que más les interesa a los agricultores cosechar, seguido del cilantro con $79 \%$ y la coliflor $37 \%$ de agricultores que cultivan cada hortaliza. Se identificó que los canales de comercialización de las hortalizas se realizan a través de dos medios intermediarios y Corabastos, siendo el canal de preferencia el de los intermediarios pues el $83.3 \%$ de los agricultores que cultivan lechuga la venden a intermediarios y tan solo el 16,6 $\%$ lo comercializa por cuenta propia hacia Corabastos; la misma tendencia se presenta con el Brócoli, el Coliflor y el cilantro, a diferencia de la remolacha la cual el $63 \%$ de los productores prefieren venderla directamente a Corabastos. Además, se identificó que de los cinco productos involucrados en el estudio la hortaliza que presenta menor variación en el precio es la lechuga, pues puede llegar a ser vendida en $\$ 3.083$ y máximo en $\$ 14.278$ pesos, y el producto que más variación presenta en su precio de venta es la remolacha ya que llega a ser vendida a $\$ 17,143$ y máximo en $\$ 98,750$.

El modelo que se planteó permite diseñar una cadena de suministro hortícola tomando decisiones en cuanto a los nodos que se deben utilizar para realizar el abastecimiento a diferentes puntos de demanda. Específicamente permite decidir si se debe abrir o no puntos de Cross docking y centros de distribución, a su vez permite establecer la cantidad de vehículos, así como su capacidad para realizar el transporte de producto entre los diferentes nodos de la cadena. Es importante resaltar que el objetivo del modelo es buscar el máximo beneficio para los productores medido en términos de utilidad, esto no quiere decir que no se tengan en cuenta otras variables consideradas de interés, como lo es el caso del impacto ambiental, el cual es medido a partir de las emisiones de $\mathrm{Co} 2$ generadas por los vehículos que se utilicen en el abastecimiento. Cabe resaltar que le 
modelo no es bi-objetivo, simplemente se tiene en cuenta las emisiones de Co2 durante el análisis y selección de la cadena de suministro que más convenga a los productores.

De acuerdo con los resultados obtenidos se estableció que la configuración más robusta en términos de utilidad es la configuración c5 y la configuración más robusta en términos de Co2 es la configuración c3; sin embargo, se estableció que la configuración de cadena de suministro hortícola bajo la cual deben operar los productores es la configuración cinco esto soportado a que el objetivo inicial del estudio era establecer la configuración que genera mayor utilidad para los productores. Cabe resaltar que en términos operaciones las configuraciones que más convenían son C3, C4 y C5. por lo tanto, la configuración C5 es adecuada en términos de operación dado que es una de las que mayor porcentaje de utilización presenta en los puntos de Cross docking, centros de distribución y vehículos.

\section{RECOMENDACIONES}

Para futuras investigaciones se identificaron las siguientes recomendaciones:

En primer lugar el análisis de robustez que se realizó se hizo midiendo la utilidad y las emisiones de Co2, lo cual permitió determinar una cadena de suministro robusta para los productores hortícolas, sin embargo podrían obtenerse resultados interesantes si se evaluaran otros indicadores para determinar la robustez estos podrían ser, valor presente neto (VAN) y tasa interna de retorno (TIR), esto debido a que el diseño está evaluando cuales son los nodos que se deben abrir y a su vez el número de vehículos que se deben adquirir para realizar el abastecimiento lo cual representa una inversión importante para los productores y sería bueno evaluarlos a partir de estos dos indicadores.

En segundo lugar, el estudio se realizó únicamente con productores pertenecientes al municipio de Cajicá, sería interesante repetir el estudio incluyendo dentro de los análisis productores hortícolas de otros municipios debido a que esto podría afectar los escenarios de estudio que se plantean inicialmente, cabe resaltar que los escenarios que se plantearon solo se tuvo en cuenta variaciones en niveles de oferta y demanda a partir de la información suministrada por los productores que participaron en el estudio, no se tuvo en cuenta el comportamiento de la producción hortícola a nivel nacional por tanto esto sería un factor importante para establecer una configuración de cadena diferentes.

Partiendo la recomendación anterior, al momento de establecer los escenarios de estudio sería bueno determinar la probabilidad de ocurrencia de estos, dado que en el presente estudio no se hizo un análisis previo para determinar tal ocurrencia. Se definieron los escenarios como si todos tuvieran la misma probabilidad de ocurrir.

\section{REFERENCIAS BIBLIOGRÁFICAS}

[1] Ali, S., Maciejewski, A., Siegel, H., \& Kim, J. (2004). Measuring the Robustness of a Resource Allocation. IEEE Transactions on Parallel and Distributed Systems, 15, 630 641. doi:10.1109/TPDS.2004.24

[2]Bello, W., \& Jairo, G. (2018). Entrevista realizada a productores horticolas del municipio de Cajicà. (M. Jimenez, Entrevistador)

[3]Departamento Administrativo Nacional de Estadísticas, D. d. (2016). Fondo Nacional de fomento Hortifruticola. Bogota: Asofrucol.

[4]Lee, \& Yu. (1997). Capacidad de planificación táctica de múltiples instalaciones con diseño experimental. HAL, archives-ouvertes, 7-10.

[5]MINAGRICULTURA. (2002). Censo Hortícola. Sabana de Bogota: Asofrucol.

[6]MINAGRICULTURA. (2016). Evaluaciones Agropecuarias del Ministerio de agricultura y desarrollo rural. Bogota D.C.: DANE.

[7]Santoso, T., Ahmed, S., Goetschalckx, M., \& Shapiro, A. (2005). A stochastic programming approach for supply chain network design under uncertainty. European Journal of Operational Research, 167, 96115. doi:0.1016/j.ejor.2004.01.046

[8]Secretaria De Ambiente y Desarrollo Rural, Alcaldia Municipal De Cajicá. (2018). Respuesta Derecho de peticion No . 51172018. Cajicá.

[9]Senior, A. (2010). Plan Horticola Nacional. Bogota: Dane.

[10]Tang, C. (2006). Robust strategies for mitigating supply chain disruptions. International Journal of Logistics Research and Applications, 33-45. doi:10.1080/13675560500405584

[11]Tordecilla Madera, R., \& Leonardo, G. (2012). Aplicación y evaluación de una metodología 
basada en el procedimiento FePIA para caracterizar la relación robustez-costo en el problema de planeación de la capacidad y localización de almacenes en cadenas de suministro. Chia. Obtenido de https://intellectum.unisabana.edu.co/bitstrea m/handle/10818/3917/Rafael\%20David\%20 Tordecilla\%20Madera.pdf?sequence=1\&isAl lowed=y

[12]Tordecilla, R., Polo, A., Muñoz, D., \& González, L. (2016). A robust design for a Colombian dairy cooperative's milk storage and refrigeration logistics system using binary programming. Elsevier, 0925-5273.

[13]Van, L., \& Vanmaele. (2002). Planificación robusta: un nuevo paradigma para la planificación de la cadena de demanda. Gestion de operaciones, 769-783.

[14]Vianchá, Z. (2014). Modelos y configuraciones de cadenas de suministro en productos perecederos. Barranquilla: ISSN.

[15]Vlajic, J., Van der Vorst, J., \& Hendrix, E. (2009). On robustness in food supply chain networks. En Towards Effective Food Supply Chains (págs. 63-82). Netherlands: Wageningen Academic. doi:10.3921/978-90-8686-705-9

[16]Wieland, A., \& Wallenburg, C. M. (2013). The influence of relational competencies on supply chain resilience: a relational view. International Journal of Physical Distribution \& Logistics Management, 300-320. doi:10.1108/JJPDLM-08-2012-0243 